\title{
Models for microtubule cargo transport coupling the Langevin equation to stochastic stepping motor dynamics: Caring about fluctuations
}

\author{
Sebastián Bouzat* \\ Consejo Nacional de Investigaciones Científicas y Técnicas, Centro Atómico Bariloche (CNEA), (8400) Bariloche, Río Negro, Argentina
}

(Received 16 July 2015; revised manuscript received 1 December 2015; published 4 January 2016)

\begin{abstract}
One-dimensional models coupling a Langevin equation for the cargo position to stochastic stepping dynamics for the motors constitute a relevant framework for analyzing multiple-motor microtubule transport. In this work we explore the consistence of these models focusing on the effects of the thermal noise. We study how to define consistent stepping and detachment rates for the motors as functions of the local forces acting on them in such a way that the cargo velocity and run-time match previously specified functions of the external load, which are set on the base of experimental results. We show that due to the influence of the thermal fluctuations this is not a trivial problem, even for the single-motor case. As a solution, we propose a motor stepping dynamics which considers memory on the motor force. This model leads to better results for single-motor transport than the approaches previously considered in the literature. Moreover, it gives a much better prediction for the stall force of the two-motor case, highly compatible with the experimental findings. We also analyze the fast fluctuations of the cargo position and the influence of the viscosity, comparing the proposed model to the standard one, and we show how the differences on the single-motor dynamics propagate to the multiple motor situations. Finally, we find that the one-dimensional character of the models impede an appropriate description of the fast fluctuations of the cargo position at small loads. We show how this problem can be solved by considering two-dimensional models.
\end{abstract}

DOI: 10.1103/PhysRevE.93.012401

\section{INTRODUCTION}

Microtubules are polymeric filaments along which the motor proteins kinesin and dynein translocate (or walk) carrying different types of cargoes in the cytoplasm. These includes lipid droplets, endosomes, mitochondria, and organelles of various types [1-3]. In order to generate motion against the large viscous drags observed inside cells, the motors use the energy from the hydrolysis of adenosine triphosphate (ATP). Microtubules are polar structures that have a plus end and a minus end. Kinesin motors move toward the plus end, while dyneins walk in the opposite direction.

Usually, the transport of a single cargo is mediated by several motors [2-5]. In the case that both kinesin and dynein motors participate, the transport is bidirectional [2-5]. This means that the organelle can move back and forth on the filament [4-6]. The way in which the bidirectional transport is coordinated is system dependent and constitutes today a subject of very active research [3,7]. Local stochastic effects as well as regulatory mechanisms can be involved $[3,6,7]$.

Mathematical models and numerical simulations play an important role in the understanding of the complex phenomena associated to multiple motor transport. This is so because most experiments provide access only to the cargo motion, while the motors' dynamics and action remain hidden. Many mathematical approaches have been used to model multiplemotor transport in different contexts and with different degree of detail. Among the most remarkable, we can mention those in $[5,8,9]$, which consider even load sharing by motors of the same species during organelle transport, and the models in [4,10-13] which consider uneven load sharing. Comparison

*bouzat@cab.cnea.gov.ar between both types of models have been provided in [10] and [14]. Other relevant models for multiple motor motion concern applications to experiments of transport of endosomes [15] and human adenovirus [16], and to the general problem of traffic on microtubules [17,18]. The contributions in [19-21] also provide important discussion on bidirectional transport by multiple motors. In this work we analyze a special class of models here referred to as the Langevin-Monte Carlo (LMC) models $[4,6,10,22]$ and propose an improved version. The LMC models consider a Langevin dynamics for the cargo coupled to a stochastic discrete-stepping dynamics for the motors ruled by a Monte Carlo algorithm. Several versions of the LMC models $[10,14,22]$ including three-dimensional formulations [23,24], as well as applications to the analysis of particular in vitro $[6]$ and in vivo $[6,25,26]$ experiments, have been developed.

The LMC models constitute a relevant framework for analyzing multiple-motor transport due to their easy implementation and to the following characteristics. First, the models consider separate descriptions for individual motors with independent cargo-motor elastic linkers. Thus the whole complexity of the cargo and motor motion, attachment, detachment, and force generation processes can be captured in a relatively simple way. Second, the Langevin dynamics for the cargo enables the analysis of the influence of the cargo size, the viscosity of the medium and the thermal fluctuations. Moreover, by considering appropriate generalizations of the Langevin equation, the basic one-dimensional model can be extended to three dimensions including rotations of the cargo $[23,24]$, and also to the case of viscoelastic media, as was done in [26] on base of the developments in [27-29]. The analysis of the fluctuations of the motion of organelles in vivo is of relevance for the characterization of the rheological properties of the cellular medium [30-33] and the elastic properties of the motor linkers as well [34]. 

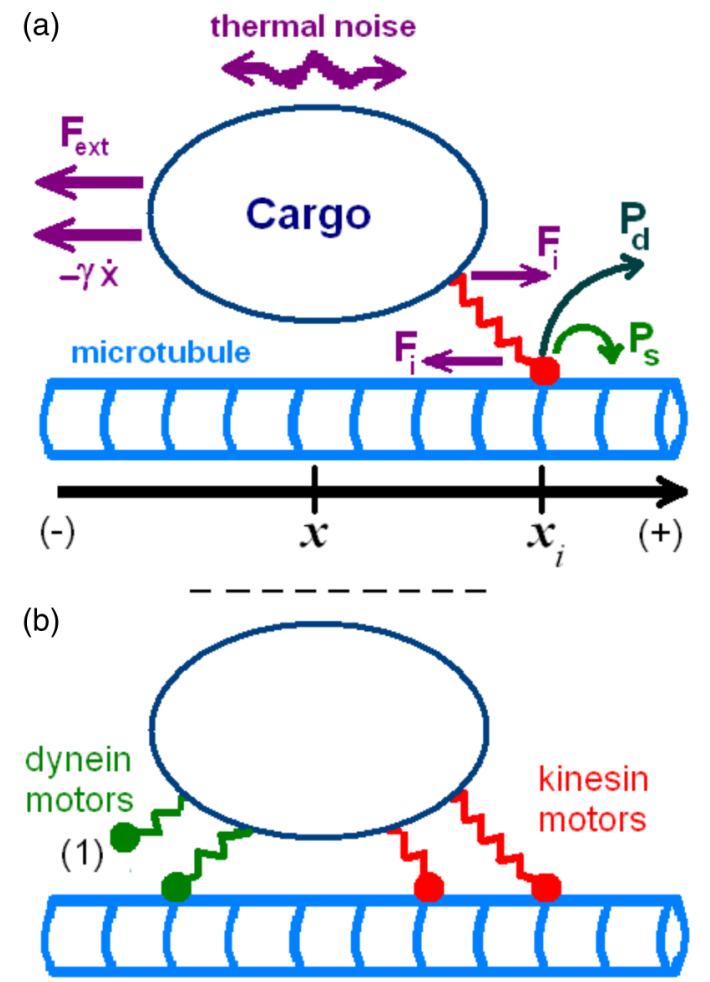

FIG. 1. Sketchs of the models. (a) Sketch of the single motor model. The motor is represented as a spring which links the cargo to the microtubule. The model is one dimensional with $x$ the cargo position and $x_{i}$ the motor position (i.e., the position where the motor binds the microtubule). The cargo dynamics is continuous and determined by the action of the external force $F_{\text {ext }}$ together with the viscous force $\gamma \dot{x}(t)$, the force exerted by the motor $F_{i}$, and the thermal noise $\xi(t)$. The motor dynamics is discrete; the allowed motor positions are represented by arches on the microtubule. The motor force $F_{i}$ determines the stepping and detachment probabilities of the motor called $P_{s}\left(F_{i}\right)$ and $P_{d}\left(F_{i}\right)$, respectively. The force $F_{i}$ is defined as a function of $x$ and $x_{i}$ in Eq. (3); this corresponds to a nonlinear interaction with a stiffness $k$ associated. (b) Sketch of the multiple motor model. Kinesin motors pull the cargo to the right (plus-end), while dynein motors pull to the left (minus-end). Each motor may be either attached to the microbutule or detached [this is the case of the dynein motor indicated as (1) in the figure]. Each attached motor exerts a force $F_{i}$ which affects its stepping and detachment probabilities as indicated in panel (a). Each detached motor has a probability per time unit $\Pi$ of reattachment.

To dwell in the subject of our work we briefly introduce the basic assumptions of the one-dimensional LMC models for cargo transport by a single motor. The general picture of the system is sketched in Fig. 1(a). The cargo motion follows the Langevin equation

$$
\gamma \frac{d x}{d t}=-F_{\mathrm{ext}}+F_{i}+\xi .
$$

Here, $x$ is the position of the cargo along the microtubule, $\gamma$ is the viscosity coefficient, $F_{\text {ext }}$ is a constant external force representing the action of an optical trap, and $\xi$ is the Gaussian thermal noise satisfying the fluctuation-dissipation relation $\left\langle\xi(t) \xi\left(t^{\prime}\right)\right\rangle=2 k_{B} T \gamma \delta\left(t-t^{\prime}\right)$, with $k_{B} T=4.1 \mathrm{pN} \mathrm{nm}$.
Finally, $F_{i}$ is the force exerted by the motor. This force is given by a nonlinear function of the cargo position $x$ and the motor position $x_{i}$ which models the elastic interaction provided by the motor stalk [see Fig. 1(a)]. The functional form $F_{i}\left(x, x_{i}\right)$ will be specified later. The allowed motor positions are discrete sites separated by a distance $\Delta x$, which are represented as vertical arches on the microtubule in Fig. 1(a). The dynamics of the motor is defined by the stepping and detachment rates, which depend on $F_{i}$. We call them $P_{s}\left(F_{i}\right)$ and $P_{d}\left(F_{i}\right)$, respectively, so that the motor steps a distance $\Delta x$ with a probability per time unit equal to $P_{s}\left(F_{i}\right)$ and detaches with probability $P_{d}\left(F_{i}\right)$. Throughout the work we set $\Delta x=8 \mathrm{~nm}$, according to the kinesin step.

In this paper we analyze the role of the thermal fluctuations in the LMC models, and their interplay with the Monte Carlo rules for the motor dynamics. In particular, we study the problem of how to provide consistent definitions for the stepping and detachment rates of individual motors as functions of the local force $F_{i}$, such that the cargo mean velocity and detachment time match previously specified (i.e., experimentally based) functions of $F_{\text {ext }}$ for the case of single motor transport. This is an open problem which deserves considerable attention since the appropriate description of single motors is essential for an accurate modeling of multiple motor transport, as we show in the section concerning multiple motors. The main difficulty comes from the fact that the experiments on single-motor transport give access to the stepping and detachment rates as functions of the external force $F_{\text {ext }}$, not as functions of the local force $F_{i}$. Clearly, due to the thermal noise and the viscous force, $F_{\text {ext }}$ and $F_{i}$ are not expected to be the same at every time. Even more, in general, the time average of $F_{i}$ is not expected to be equal to $F_{\text {ext }}$. Along our work we analyze the validity of the usual approximations for $P_{s}\left(F_{i}\right)$ and $P_{d}\left(F_{i}\right)$ found in the literature, and we propose a modified model that includes a dependence on the recent history of the local force. As we will show, this proposal enhances remarkably the performance of the LMC models and provides a solution for the problem of finding consistent definitions for the stepping and detachment rates.

The Langevin equation [35] has been used for describing transport driven by molecular motors since the pioneering works on ratchets models in the 1990s [36,37]. Many methods for resolution of the Langevin equation has been considered for this and other applications in physics and chemistry [35], including path integrals techniques [38], mapping to Fokker-Planck equations, and spectral methods [35,39]. In the case of the LMC models, the coupling with the Monte Carlo dynamics for the motors makes preferably a numerical treatment of the Langevin equation based on a standard time discretization scheme $[4,6,10,22]$.

The paper is organized as follows. In the next section we introduce the models that we study along the work. Namely, a standard LMC model, a zero temperature model developed for the sake of comparison, and our proposal. We also explain the way in which simulations are performed and the quantities we analyze. In the section Results and Analysis we analyze and compare the models for different single and multiple-motor situations. The last section contains our main conclusions and additional discussion. 


\section{MODELS, SIMULATIONS AND RELEVANT QUANTITIES}

\section{A. Standard model coupling the Langevin cargo dynamics to Monte Carlo-ruled motor stepping}

As stated in the Introduction, several LMC models have been developed in the literature. In this work we focus on the one-dimensional version used in $[6,10]$ and [25], which from now on is referred to as standard Langevin-Monte Carlo model (SLMC). At the end of this section we briefly explain the similarities and differences with other LMC models, some of which are analyzed in the final part of the paper.

The SLMC model considers the cargo as linked permanently to $N_{f}$ forward motors (kinesins) and $N_{b}$ backward motors (dyneins), as sketched in Fig. 1(b). The motion of the organelle follows the Langevin equation

$$
\gamma \frac{d x}{d t}=-F_{\mathrm{ext}}+\sum_{i} F_{i}+\xi .
$$

Here, $F_{i}$ with $i=1, \ldots, N_{f}+N_{b}$ is the force exerted by the $i$ th motor, while $F_{\text {ext }}$ is the external force and $\xi$ the thermal noise as explained for the single motor case of Eq. (1). The constant $\gamma$ is calculated using the Stokes formula [4,10,22] $\gamma=6 \pi R \eta$, with $R$ the radius of the cargo and $\eta$ the viscosity of the medium. Throughout the paper we fix $R=500 \mathrm{~nm}$ and we consider the ratio $n_{w}=\eta / \eta_{w}$, with $\eta_{w}=9.1 \times 10^{-10} \mathrm{pN} \mathrm{s} / \mathrm{nm}^{2}$ the viscosity of water, as the relevant parameter which quantifies the viscosity.

At a given time, each motor can be either attached to the microtubule or detached [see Fig. 1(b)]. For an attached motor, the motor position $x_{i}$ can be interpreted as the center of mass of the motorheads. The allowed motor positions are the discrete sites $x_{j}=j \Delta x$ with integer $j$ and $\Delta x=8 \mathrm{~nm}$. Following the assumptions in $[4,6,10,14,22-25]$, each attached motor can exert only attractive forces on the cargo provided that a critical distance $x_{r}$ associated to the motor length is surpassed. The force $F_{i}$ is defined as

$$
F_{i}=F_{i}\left(x, x_{i}\right)= \begin{cases}0 & \text { for }\left|x-x_{i}\right| \leqslant x_{r}, \\ k\left(x_{i}-x-x_{r}\right) & \text { for }\left(x_{i}-x\right)>x_{r} \\ -k\left(x-x_{i}-x_{r}\right) & \text { for }\left(x-x_{i}\right)>x_{r}\end{cases}
$$

where $k$ is the stiffness of the $i$ th motor. For a detached motor, the position $x_{i}$ is irrelevant and we set $F_{i}=0$.

Each motor has a stepping probability per time unit $P_{s}\left(F_{i}\right)$ and a detachment probability $P_{d}\left(F_{i}\right)$. The steps are of absolute length $\Delta x=8 \mathrm{~nm}$. Kinesins advance in the positive direction while dyneins advance in the negative one. Along the work we consider functional forms for $P_{s}\left(F_{i}\right)$ and a detachment probability $P_{d}\left(F_{i}\right)$ valid for different motor types and conditions which are given in Table I. Note that no backward steps are allowed in the SLMC model, i.e., neither negative steps for kinesins nor positive steps for dyneins (the case of a model with back-stepping is at the end of the paper). Finally, each detached motor has a constant probability per time unit $\Pi$ of attaching to the microtubule. The attachment occurs with equal probability in any of the sites $x_{j}=j \Delta x$ with integer $j$ satisfying $\left|x-x_{j}\right|<x_{r}$ (i.e., with the motor-linker relaxed).

To compute the evolution of the cargo-motor system, the time is discretized in steps of duration $d t$ which must be taken much smaller than the relaxation time of the motor springs $\tau_{k}=\gamma / k$. Typically we consider $d t \sim 10^{-6}$ s or lower, depending on the viscosity. Then, the motor dynamics is evolved together with a standard discrete-time approximation for Eq. (2). The details of the algorithm are given in Appendix A.

The differences between the SLMC and other LMC models involve mainly two aspects. Namely, the details of the Monte Carlo rules for the motor dynamics and the dimensionality of the Langevin equation. For instance, Ref. [4] uses the same Langevin equation but considers only kinesin motors ruled by a more detailed stepping algorithm which includes two stages of the chemical cycle. References [14,22] also consider the one-dimensional Langevin formulation but with a Monte Carlo algorithm which include motor backstepping. Meanwhile, the works in [23] and [24] consider three-dimensional versions of the Langevin equation with the motor dynamics set as in the SLMC and as in the model in [4], respectively. In some works $[4,6]$, only the discrete-time version of the Langevin equation is introduced.

\section{B. Zero temperature model}

In order to analyze the influence of the thermal fluctuations on the SLMC model, we compare the results with those from a zero temperature model (ZT) which considers Eq. (2) with

TABLE I. Parameters and functional forms for the single-motor dynamics. Data taken from [6]. The functional form for $P_{s}(F)$ is also considered in other works [10,23,25,26], although with different values of $v_{0}, w$, and $F_{0}$. In all the cases $\Delta x$ is set equal to $8 \mathrm{~nm}$, the units of

\begin{tabular}{|c|c|c|c|}
\hline & kinesin (in vitro) & kinesin (in vivo) & dynein (in vivo) \\
\hline$P_{s}(F)$ for $F<0$ & & $v_{0} / \Delta x$ & \\
\hline$P_{s}(F)$ for $0 \leqslant F \leqslant F_{0}$ & & $v_{0}\left[1-\left(F / F_{0}\right)^{u}\right.$ & \\
\hline$P_{s}(F)$ for $F>F_{0}$ & & 0 & \\
\hline$v_{0}$ & $1000 \mathrm{~nm} / \mathrm{s}$ & $520 \mathrm{~nm} / \mathrm{s}$ & $800 \mathrm{~nm} / \mathrm{s}$ \\
\hline$F_{0}$ & $5 \mathrm{pN}$ & $2.5 \mathrm{pN}$ & $2.5 \mathrm{pN}$ \\
\hline$w$ & 2 & 2 & 0.5 \\
\hline$P_{d}(F)$ for $|F| \leqslant F_{0}$ & $\exp (|F| / 4)$ & $0.35 \exp (|F| / 2)$ & $0.37 \exp (|F| / 1.74)$ \\
\hline$P_{d}(F)$ for $|F|>F_{0}$ & $(1.07+0.186|F|)$ & $(1.535+0.186|F|)$ & {$[0.254(1-\exp (-|F| / 1.97))]^{-1}$} \\
\hline$\Pi$ & not used & $1.1 \mathrm{~s}^{-1}$ & $1.29 \mathrm{~s}^{-1}$ \\
\hline$k$ & $0.32 \mathrm{pN} / \mathrm{nm}$ & $0.32 \mathrm{pN} / \mathrm{nm}$ & $0.32 \mathrm{pN} / \mathrm{nm}$ \\
\hline$n_{w}$ & 1 (water viscosity) & 10 & 10 \\
\hline
\end{tabular}
$P_{d}(F)$ are $\mathrm{s}^{-1}$, and the formulas for $P_{d}(F)$ assume that $F$ is expressed in $\mathrm{pN}$. 
vanishing thermal noise. The motor dynamics remains the same as in the SLMC model so that the motors are assumed to work at normal physiological conditions. The detailed algorithm is given in Appendix A.

\section{Model with time averaged forces}

The SLMC model assumes that the stepping and detachment rates are well defined functions of the instantaneous (i.e., fast fluctuating) force $F_{i}$ acting on the motor domain. As we will show, this approximation leads to some problems on the reproduction of the experimental results for single motors, especially when the observed mean run-time is a nonmonotonous function of $F_{\text {ext }}$. To overcome this problem we propose an alternative model for the motor dynamics that will be referred to as the time-averaged forces model (TAF). At this point it is necessary to write down explicitly the dependences of the positions and forces on the time $t$ to further develop the model. Thus we write $x(t)$ and $x_{i}(t)$ for the cargo and motor positions, respectively, and $F_{i}\left(x(t), x_{i}(t)\right)$ for the forces determined by the relation given in Eq. (3).

We consider that the cargo dynamics obeys Eq. (2) as in the SLMC, while the motor stepping dynamics responds not to the instantaneous values of the forces $F_{i}\left(x(t), x_{i}(t)\right)$ but to the following time-averaged local forces:

$$
\tilde{F}_{i}(t)=\frac{1}{\tau_{m}} \int_{0}^{t} F_{i}\left(x\left(t-t^{\prime}\right), x_{i}\left(t-t^{\prime}\right)\right) \exp \left(-t^{\prime} / \tau_{m}\right) d t^{\prime}
$$

Due to the exponential weight, the average involves essentially a time window of duration $\tau_{m}$ on the recent history of the force. The condition $t \gg \tau_{m}$ leading to $\frac{1}{\tau_{m}} \int_{0}^{t} \exp \left(-t^{\prime} / \tau_{m}\right) d t^{\prime} \simeq 1$ is required for a meaningful definition of the average.

Other choices of the weight function could lead to similar results for the system dynamics; however, the exponential weight enables us to write the following differential equation for $\tilde{F}_{i}(t)$ :

$$
\frac{d \tilde{F}_{i}}{d t}(t)=-\frac{1}{\tau_{m}}\left[\tilde{F}_{i}(t)-F_{i}\left(x(t), x_{i}(t)\right)\right] .
$$

This can be obtained by simple derivation of Eq. (4) and integration by parts.

Hence the TAF model considers Eqs. (2) and (5), which in the compact notation read

$$
\begin{gathered}
\gamma \frac{d x}{d t}=-F_{\mathrm{ext}}+\sum_{i} F_{i}+\xi, \\
\frac{d \tilde{F}_{i}}{d t}=-\frac{1}{\tau_{m}}\left[\tilde{F}_{i}-F_{i}\right],
\end{gathered}
$$

coupled to the discrete motor dynamics ruled by the time averaged forces $\tilde{F}_{i}$. This means the same Monte Carlo algorithm introduced for the SLMC but substituting $P_{S}\left(F_{i}\right)$ and $P_{d}\left(F_{i}\right)$ by $P_{s}\left(\tilde{F}_{i}\right)$ and $P_{d}\left(\tilde{F}_{i}\right)$, respectively. The details of the algorithm are given in Appendix A. It is worth remarking that while the motors obey to the averaged forces, the cargo is still driven by the instantaneous fast-fluctuating forces $F_{i}$ defined in Eq. (3), since this is the appropriate assumption for the Langevin dynamics and important for reproducing the cargo motion at small time scales. Note that these considerations do not lead to inconsistency, as the dynamical models for motor and cargo are essentially different from each other, given that one is discrete and probabilistic, while the other is continuous both in time and space. The introduction of time averaged forces for the motor dynamics corresponds to a coarse graining treatment of the force which, as we will show, improves the results for cargo motion.

Importantly, the idea that the stepping and detachment rates may be defined in terms of time averaged forces or, equivalently, considered as dependent on the recent history of the force, is reasonable from various points of views. First, note that both for kinesin and dynein the time between steps (i.e., the duration of the associated chemical cycles) is of the order of $10^{-2} \mathrm{~s}$. The cycles involve several stages whose durations may depend on the local force in different manners $[4,40]$. Thus the duration of each cycle would be affected by the history of the force along the whole cycle. Given that we are not going to describe the details of the different reactions within the cycle but assume instead a single stepping probability, it is reasonable to consider the motor-force averaged on a time window of duration $\tau_{m} \sim 10^{-2} \mathrm{~s}$. Similarly, given that detachment is not equally probable along the chemical cycle [4] and that we are not going to describe the details of its time dependence, it is reasonable to consider the detachment rate as dependent on an average force acting on the mentioned time scale.

From another point of view, note that if the function $P_{s}(F)$ [or $P_{d}(F)$ ] represented for instance a Kramers rate derived from a microscopic Langevin description of the motion of the motor heads, the force $F$ could not correspond to the instantaneous fast-fluctuating local force $F_{i}$. Instead, it should be a parameter which must remain essentially constant on the time scale relevant for the transition. More specifically, a Langevin description of the motion of the motor heads could consider the local force $F_{i}\left(x(t), x_{i}(t)\right)$ as given by $F_{i}\left(x(t), x_{i}(t)\right)=\left\langle F_{i}\left(x(t), x_{i}(t)\right)\right\rangle+\delta F_{i}(t)$, with $\left\langle F_{i}\left(x(t), x_{i}(t)\right)\right\rangle$ the mean value of the force during a time window relevant for the transition and $\delta F_{i}(t)$ a fast fluctuating quantity with zero mean. Then, the calculation of the Kramers rate would average out the fluctuations and lead to a transition probability dependent only on $\left\langle F_{i}\left(x(t), x_{i}(t)\right)\right\rangle$.

From a mathematical point of view, the definition of the stepping and detachment rates in terms of the averaged forces implies the introduction of memory in the motor dynamics. This is because, at a given time, the rates are conditioned not only by the current value of the force but also by the values attained previously. As justified before, a time window of the order of the time between steps will be considered for averaging. Thus the memory will be relevant on such time scale. Interestingly, although with a different formalization, the existence of memory effects in the motor motion was recently considered in [21].

\section{Simulations: Mean velocities and detachment rates}

For the three models (SLMC, ZT, and TAF), the simulations of single motor transport are made as follows. A constant value of $F_{\text {ext }}$ is considered. As initial condition, at $t=0$, the motor is attached to the microtubule in one of the sites $x_{j}=j \Delta x$ satisfying $\left|x-x_{j}\right|<x_{r}$. Then, as in [4], a small transient time $t_{t r}$ is skipped (typically $t_{t r} \sim 0.1 \mathrm{~s}$ ) to filter out the initial backward motion of the cargo caused by the action 
of $F_{\text {ext }}$ when the motor-linker is not stretched. During such transient, detachment is not allowed. For $t>t_{t r}$ detachment is allowed, and the simulation dynamics continues until the motor detaches. The time at which the motor detaches is called $t_{d}$. The mean velocity of the run is then defined as $v_{\text {run }}=\left(x\left(t_{d}\right)-x\left(t_{r}\right)\right) /\left(t_{d}-t_{t r}\right)$, while the run time is $T_{\text {run }}=$ $t_{d}-t_{t r}$. Finally, we average over realizations to define the mean cargo velocity as a function of $F_{\text {ext }}$ as $v\left(F_{\text {ext }}\right)=\left\langle v_{\text {run }}\right\rangle$ and the detachment probability $Q\left(F_{\text {ext }}\right)=1 /\left\langle T_{\text {run }}\right\rangle$. Here, \langle\rangle means average on runs computed with the same value of $F_{\text {ext }}$.

Simulations for multiple motors are performed in a completely analogous way, as explained in the section concerning multiple motor transport.

\section{RESULTS AND ANALYSIS}

\section{A. Force-velocity and force-detachment relations for single motors}

The force-velocity relations found in single-motor experiments with different motor types and setups can be reasonably well approximated as [6,10,23]

$$
v_{\text {obs }}\left(F_{\text {ext }}\right)= \begin{cases}v_{0}, & F_{\text {ext }}<0, \\ v_{0}\left[1-\left(F_{\text {ext }} / F_{S}\right)^{w}\right], & 0 \leqslant F_{\text {ext }} \leqslant F_{s}, \\ 0, & F_{\text {ext }}>F_{s} .\end{cases}
$$

Here, $v_{\text {obs }}\left(F_{\text {ext }}\right)$ indicates the observed mean velocity as a function of the external load, $F_{S}$ is the stall force leading to a vanishing velocity, $v_{0}$ is the zero-load velocity, and $w>0$ is a parameter which controls the decay of the velocity with the force. Equation (8) neglects the slow backward motor motion observed at superstall conditions $\left(F_{\text {ext }}>F_{S}\right)$ and contemplates the fact that assisting forces $\left(F_{\text {ext }}<0\right)$ produce very small effects on the motor velocity. Hence Eq. (8) can be taken as a useful phenomenological formula which approaches the results of most experiments [6,10,23], although it does not necessarily give a highly accurate description of any particular experiment for every value of $F_{\text {ext }}$. For the purpose of this work, however, $v_{\text {obs }}\left(F_{\text {ext }}\right)$ represents the results that we want to reproduce with our simulations.

Taking into account this force-velocity relation, the SLMC models in $[6,10,25]$ and also the three-dimensional model in [23] assume the stepping rate $P_{S}\left(F_{i}\right)$ as given by the formula indicated in Table I with the parameter $F_{0}$ set equal to the experimental stall force $F_{s}$. This corresponds to setting $P_{s}\left(F_{i}\right)=v_{\mathrm{obs}}\left(F_{i}\right) / \Delta x$ [41]. The assumption is reasonable since a random walker with such stepping probability would advance with mean velocity $v_{\text {obs }}\left(F_{i}\right)$ if $F_{i}$ is constant. However, this does not take into account the difference existing between the constant external force $F_{\text {ext }}$ and the fluctuating local force $F(t)$ which acts on the motor domain. In fact, as a consequence of this assumption, the mean velocity $v\left(F_{\text {ext }}\right)$ calculated from simulations results to be different to $v_{\text {obs }}\left(F_{\text {ext }}\right)$. This is shown in Fig. 2(a) where we plot $v\left(F_{\text {ext }}\right)$ calculated for the SLMC, ZT, and TAF models with the parameters indicated in Table I for kinesin in vitro, together with the corresponding $v_{\text {obs }}\left(F_{\text {ext }}\right)$. It can be seen that, for the SLMC model, the calculated stall force (approximately $6.5 \mathrm{pN}$ ) is considerably larger than the expected one $\left(F_{s}=5 \mathrm{pN}\right)$. Moreover, in the range $0.5-3 \mathrm{pN}$, the calculated velocity is smaller than $v_{\text {obs }}\left(F_{\text {ext }}\right)$. This all means
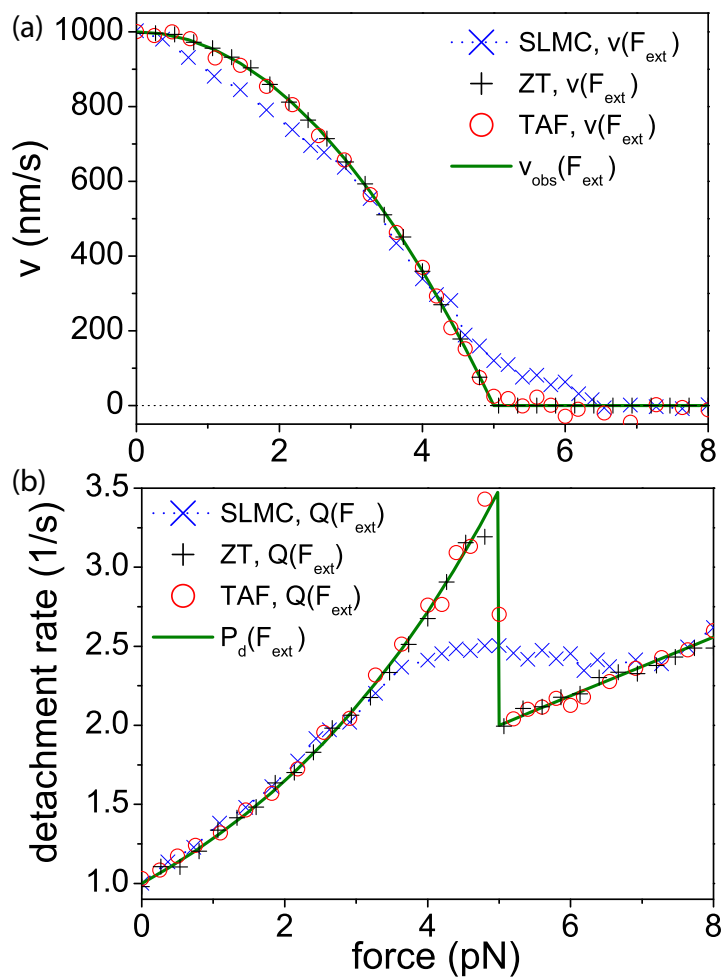

FIG. 2. Velocity and detachment rates for single-motor transport calculated with SLMC, ZT, and TAF models. Simulations considering the parameters for kinesin in vitro indicated in Table I. Panel (a) shows the results from simulations for the mean velocity $v\left(F_{\text {ext }}\right)$ for the models SLMC, ZT, and TAF, together with the expected result $v_{\text {obs }}\left(F_{\text {ext }}\right)$ given in Eq. (8). Panel (b) shows the calculated detachment rate $Q\left(F_{\text {ext }}\right)$ for the same systems together with the expected curve $P_{d}\left(F_{\text {ext }}\right)$. The results from the TAF model are for $\tau_{m}=10^{-2} \mathrm{~s}$.

that, if the experimental force-velocity relation of Eq. (8) is assumed to hold at the level of the motorheads within the SLMC model, it does not arise as the relation between the computed cargo velocity and the external force. In contrast, the TAF model with $\tau_{m}=0.01 \mathrm{~s}$ and the ZT model provide good results, since the calculated velocities match the function $v_{\text {obs }}\left(F_{\text {ext }}\right)$.

Something similar occurs with the detachment rate. The simulations analyzed in Fig. 2 consider the detachment probability of the motor as a function of the local force $\left(P_{d}\left(F_{i}\right)\right)$ defined by the experimental formula given in Table I for kinesin in vitro. Figure 2(b) shows that, while for the SLMC the calculated detachment rate $Q\left(F_{\text {ext }}\right)$ does not coincide with the expected result $P_{d}\left(F_{\text {ext }}\right)$ for every value of $F_{\text {ext }}$, the TAF and ZT models provide full consistence between these two quantities.

In Fig. 3 we show results from the SLMC, TAF, and ZT models for other relevant choices of the stepping and detachment probabilities. Namely, those corresponding to kinesin in vivo and dynein in vivo proposed in Ref. [6], and to a kinesin model with exponential detachment similar to those considered in [23] and [25]. The results indicate that, in all the cases, the problem with the SLMC model persists, while the ZT and TAF models give consistent results. However, in the case 

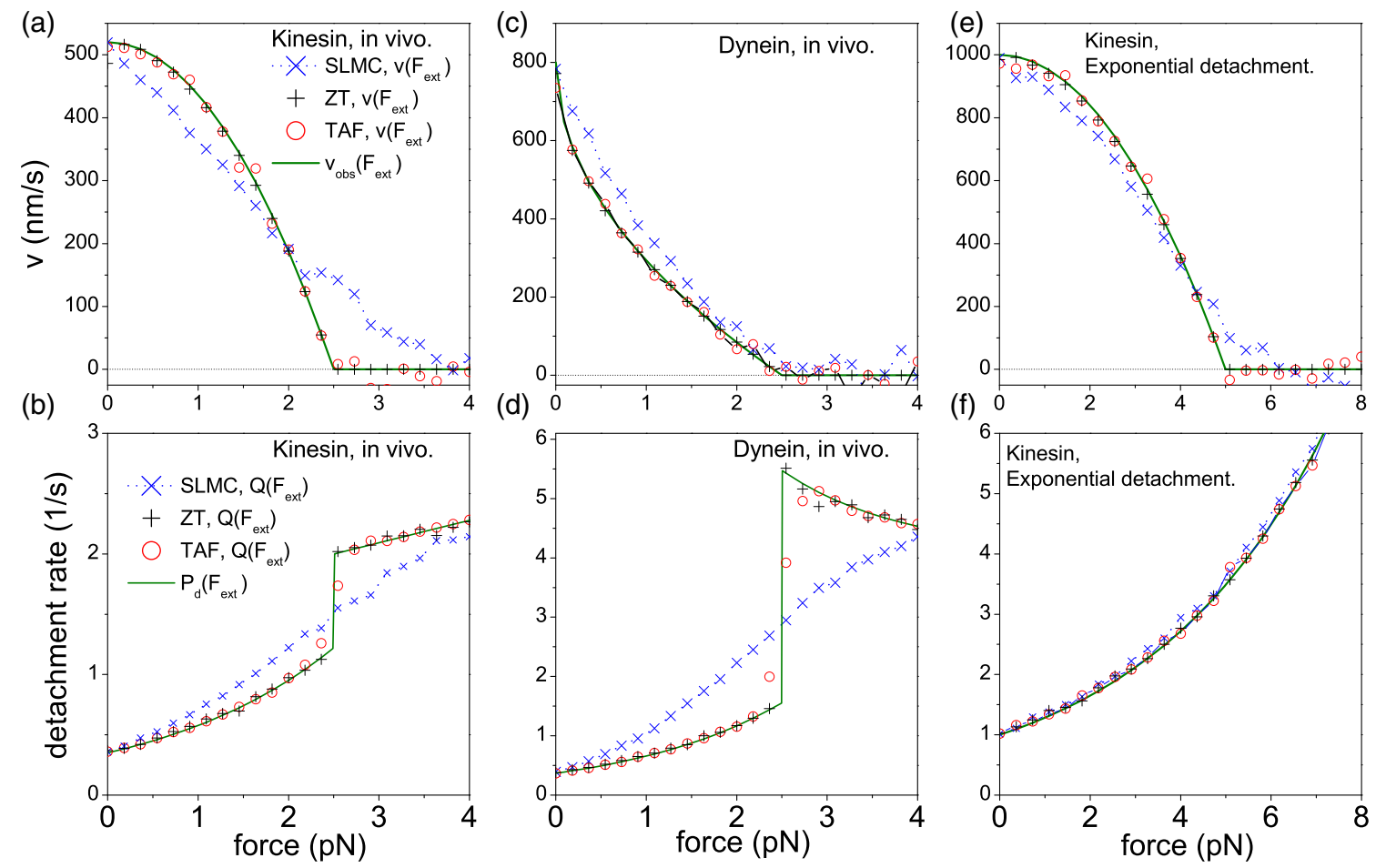

FIG. 3. Velocity and detachment rate for single motors for various motor types. Panel (a) shows the mean velocity calculated from simulations for the SLMC, ZT, and TAF models considering the parameters for kinesin in vivo indicated in Table I, together with the expected result $v_{\text {obs }}\left(F_{\text {ext }}\right)$. Panel (b) shows the results for $Q\left(F_{\text {ext }}\right)$ for the three models for the same parameters together with the expected result $P_{d}\left(F_{\text {ext }}\right)$. Panels (c) and (d) repeat the calculations in panels (a) and (b) but considering parameters for dynein in vivo from Table I. In this case, a change of sign is introduced in the velocity so that we get positive velocities for minus-end transport. Panels (e) and (f) consider the case of kinesin with exponential detachment. This corresponds to the same parameters as for kinesin in vitro but considering $P_{d}(F)=\varepsilon$ exp $\left[|F| / F_{d}\right]$ with $\varepsilon=1 \mathrm{~s}^{-1}$ and $F_{d}=4 \mathrm{pN}$. In all the cases the results from the TAF model are for $\tau_{m}=10^{-2} \mathrm{~s}$.

of kinesin with exponential detachment the deviation of the SLMC occurs only in the velocity curve.

The errors of the mean quantities plotted in Figs. 2 and 3 are significantly small. Error bars are not shown since they result of the same size as the symbols and hinder the identification of the curves. The relevance of the results becomes apparent in Fig. 4 where we analyze the distributions of time averaged velocities of single runs for some selected values of $F_{\text {ext }}$, and also distributions of run times. Figures 4(a) and 4(b) show the distributions of velocities corresponding to the calculations in Fig. 2(a) for $F_{\text {ext }}=1.8 \mathrm{pN}$ and $F_{\text {ext }}=5 \mathrm{pN}$, respectively. The differences between the distribution for the SLMC model and those for the other models are appreciable. In particular, for $F_{\text {ext }}=5 \mathrm{pN}$ (i.e., the expected stall force) the distributions for the TAF and ZT models are symmetric around $v=0$ as desired, while the one for the SLMC model is strongly shifted to positive velocities. In Figs. 4(c) to 4(f) we show velocity distributions and also distributions of runtimes for two selected values of the force corresponding to the system analyzed in Figs. 3(a) and 3(b). This means parameters for kinesin in vivo. For the sake of simplicity we only include results for SLMC and TAF. In all the cases, the differences between the TAF and SLMC models are relevant. Note that, as explained before, the calculated detachment rate plotted in Fig. 3(b) is the inverse of the mean of the distributions shown in 4(d) and 4(f), for the indicated values of $F_{\text {ext }}$.
As the only difference between the ZT and SLMC models is the consideration on the thermal noise, it becomes apparent that the problem of the SLMC for fitting the expected results in Figs. 2 and 3 should be related to the influence of the fluctuations. To understand this, we begin by focusing on the results in Fig. 2(a). First, the fact that the calculated stall force is larger than $F_{0}$ is easy to understand. Note that for $F_{\text {ext }} \geqslant F_{0}$, the thermal fluctuations can eventually push forward the cargo so that the elastic linker may relax and the local force $F_{i}$ results smaller than $F_{0}$. Thus $P_{s}\left(F_{i}\right)$ becomes nonzero and the motor may step. This means that the motor works as a ratchetlike device which can advance when helped by fluctuations. On the other hand, the fact that the cargo velocity in the range $0.5-3 \mathrm{pN}$ results lower than $v_{\text {obs }}\left(F_{\text {ext }}\right)$ is due to the thermal fluctuations leading to $F_{i}>F_{\text {ext }}$ (i.e., those which move the cargo backward). Such fluctuations cannot be compensated by the fluctuations leading to $F_{i}<F_{\text {ext }}$ since there is a maximum value of $P_{s}\left(F_{\text {ext }}\right)$. Generally speaking, due to the fluctuations, the calculated velocity curve results flatter than that for $v_{\text {obs }}\left(F_{\text {ext }}\right)$. Note that, in all the velocity curves in Figs. 2 and 3, the SLMC yields $v\left(F_{\text {ext }}\right)<v_{\text {obs }}\left(F_{\text {ext }}\right)$ in the zones where $v_{\text {obs }}\left(F_{\text {ext }}\right)$ is concave downward and $v\left(F_{\text {ext }}\right)>v_{\text {obs }}\left(F_{\text {ext }}\right)$, where $v_{\text {obs }}\left(F_{\text {ext }}\right)$ is concave upward. The differences between $Q\left(F_{\text {ext }}\right)$ and $P_{d}\left(F_{\text {ext }}\right)$ found for the SLMC model can be understood in a similar way as those for the velocity taking into account the influence of the fluctuations. The blurring 


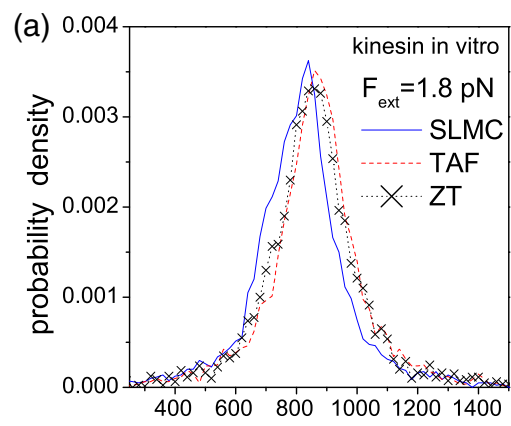

(b)

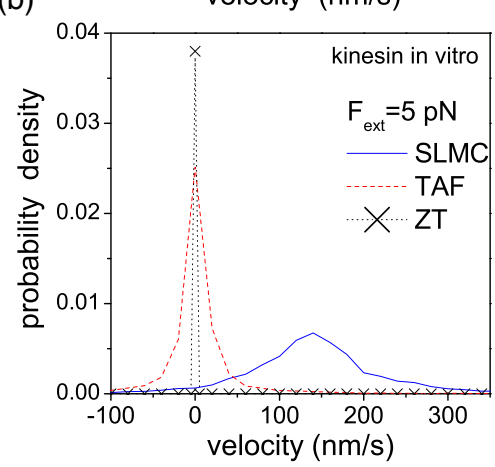

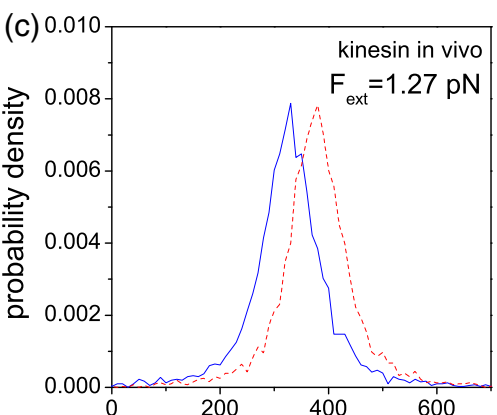

(d)

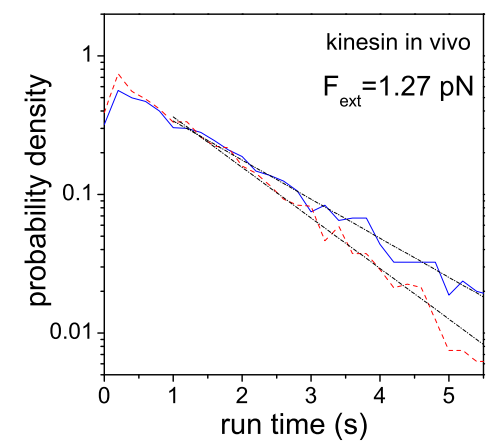

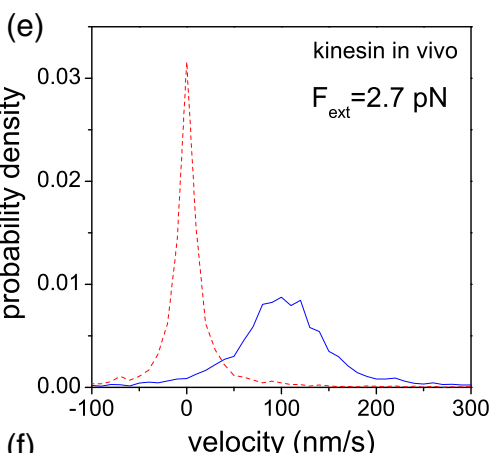

(f)

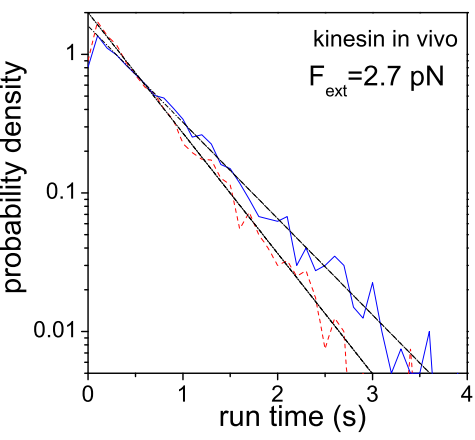

FIG. 4. Velocity distributions and run-time distributions for selected systems analyzed in Figs. 2 and 3 considering different motor models. Panels (a) and (b) show the velocity distributions for the kinesin in vitro system analyzed in Fig. 2 considering the cases $F_{\text {ext }}=1.8$ pN and $F_{\text {ext }}=5 \mathrm{pN}$ (stall force), respectively. Results for the SLMC, ZT, and TAF models are shown. Panels (c) and (e) show the velocity distributions for the kinesin in vivo system analyzed in Fig. 3 considering the cases $F_{\text {ext }}=1.27 \mathrm{pN}$ and $F_{\text {ext }}=2.7$ pN, respectively. Results for SLMC and TAF models are shown. Finally, panels (d) and (f) show the run-time distributions for the cases analyzed in panels (c) and (e), respectively. The dash-dotted lines in panels (d) and (f) correspond to exponential laws which approximate the tails of the run-time distributions. The plotted functions are proportional to $\exp (-a t)$ with $a=0.65 / s$ [panel (d), SLMC], $a=0.84 / s$ [panel (d), TAF], $a=1.65 / s$ [panel (f), SLMC], and $a=2$ [panel (f), TAF].

of the discontinuities in the detachment probabilities are expectable.

\section{B. Robustness of the TAF model against varying $\boldsymbol{\tau}_{\boldsymbol{m}}$}

In order to check the robustness of the TAF model against changes in the parameter $\tau_{m}$, we have systematically studied the mean velocity $v\left(F_{\text {ext }}\right)$ and the detachment probability $Q\left(F_{\text {ext }}\right)$ results considering different values of $\tau_{m}$ for the kinesin in vitro case. Our analysis indicates that in the range $3 \times 10^{-3} \mathrm{~s} \lesssim \tau_{m} \lesssim 3 \times 10^{-2} \mathrm{~s}$, the results for the TAF model are essentially independent of $\tau_{m}$ and agree quite well with the expected velocity and detachment probabilities. This happens in the whole relevant range of the external force. To illustrate these results, in Fig. 5 we show the mean velocity $v\left(F_{\text {ext }}\right)$ and the computed detachment probability $Q\left(F_{\text {ext }}\right)$ for the TAF model considering different values of $\tau_{m}$, and also curves of velocity as function of $\tau_{m}$ for fixed values of $F_{\text {ext }}$.

Hence, in the range $3 \times 10^{-3} \mathrm{~s} \lesssim \tau_{m} \lesssim 3 \times 10^{-2}$ s the TAF model results in a robust consistent model which reproduces the expected results. As $\tau_{m}$ decreases in the region $\tau_{m}<$ $3 \times 10^{-3} \mathrm{~s}$, the TAF model gradually approaches the SLMC model. For instance, see the case $\tau_{m}=10^{-4} \mathrm{~s}$ in Figs. 5(a) and 5(b). On the other hand, for $\tau_{m} \gtrsim 0.1 \mathrm{~s}$, the input and output probabilities also cease to agree.

The fact that the condition $3 \times 10^{-3} \mathrm{~s} \lesssim \tau_{m} \lesssim 3 \times 10^{-2} \mathrm{~s}$ leads to a matching between the calculated and expected probabilities for stepping and detachment can be understood by analyzing the dynamics at different time scales. We focus on two characteristic times. Namely, the mean time between steps $\tau_{s}$, which is typically of order $10^{-2} \mathrm{~s}$, and the relaxation time associated to the motor linker $\tau_{k}=\gamma / k$ which is smaller than $\tau_{s}$ excepting for very large viscosities $\left(n_{w}>500\right)$. For example, for a cargo of radius $250 \mathrm{~nm}$ and water viscosity we get $\tau_{k}=1.4 \times 10^{-5} \mathrm{~s}$. Now, note that the time average of the cargo velocity $\langle\dot{x}(t)\rangle$ performed on a time window of length $\tau_{m}$ would be nonzero for $\tau_{m} \lesssim \tau_{k}$ due to the coherent motion associated to the relaxation of the motor springs. Moreover, it would also be nonzero for $\tau_{m}>\tau_{s}$, since in such a time scale there is motor-driven motion. In contrast, for $\tau_{k} \ll \tau_{m} \lesssim \tau_{s}$ we expect $\langle\dot{x}(t)\rangle \simeq 0$. In this situation, the time average of the Langevin equation on the same time window leads to $\left\langle F_{i}\right\rangle \simeq F_{\text {ext }}$. Thus, for the TAF model in this region of $\tau_{m}$ we have $P_{s}\left(\tilde{F}_{i}\right) \simeq P_{s}\left(F_{\text {ext }}\right)$ and $P_{d}\left(\tilde{F}_{i}\right) \simeq P_{d}\left(F_{\text {ext }}\right)$ so that the motor dynamics responds directly to the external force and leads to a matching between the calculated and expected quantities.

In short, when presenting the TAF model we have argued why it is reasonable to consider a value of $\tau_{m}$ of the order of the time between steps. Now, our results indicate that the model is robust in the region $\tau_{k} \ll \tau_{m} \lesssim \tau_{s}$ and that for such values of $\tau_{m}$ the functions $P_{s}\left(\tilde{F}_{i}\right)$ and $P_{d}\left(\tilde{F}_{i}\right)$ can be taken directly from the experiments. Note that in our analysis we have considered $v_{\text {obs }}\left(F_{\text {ext }}\right)$ from Eq. (8) and $P_{d}\left(F_{\text {ext }}\right)$ from 

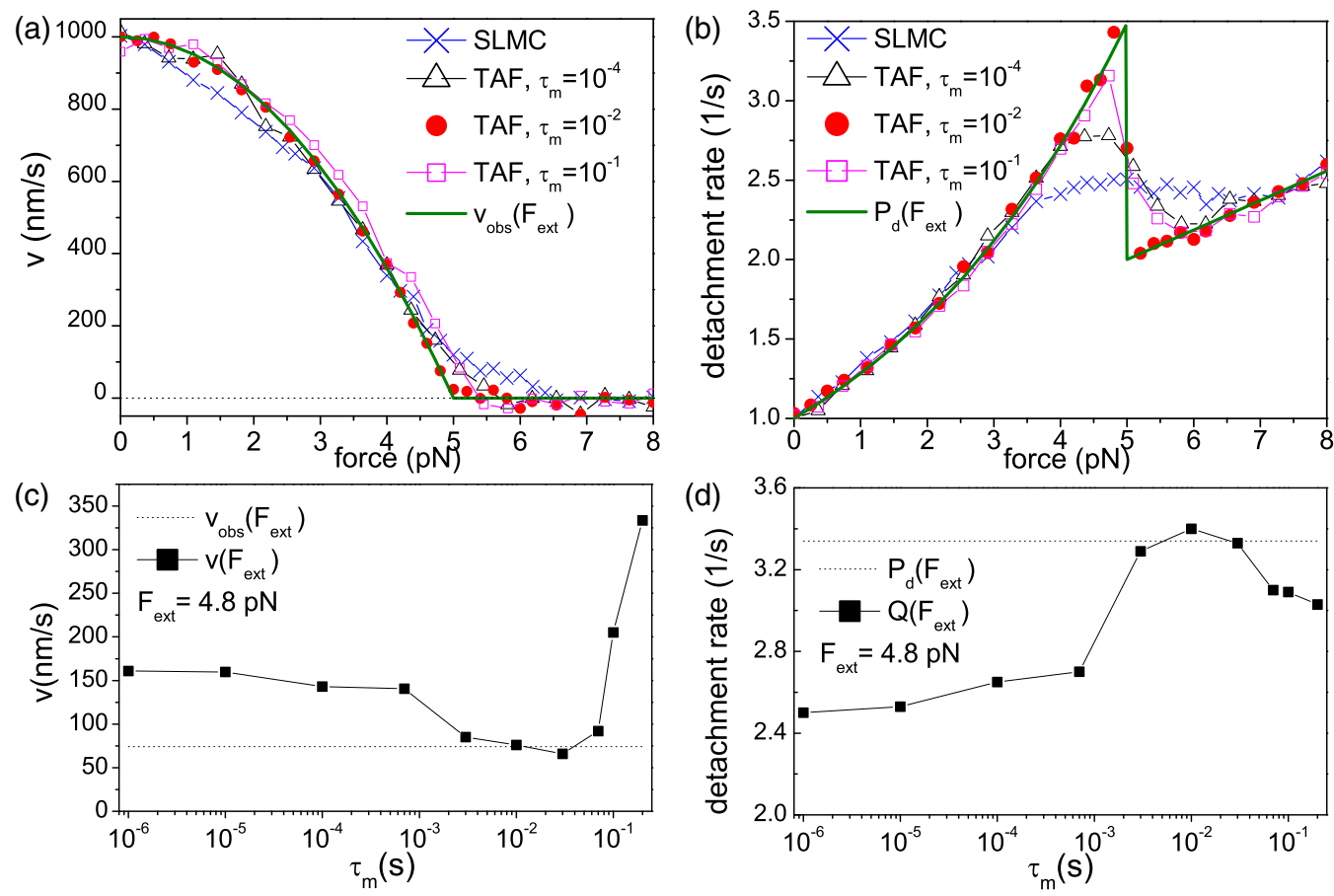

FIG. 5. TAF model for different values of $\tau_{m}$. (a) Mean velocity as a function of the external force computed with the TAF model considering different values of $\tau_{m}$, together with the results for the SLMC model and the expected result $v_{\text {obs }}\left(F_{\text {ext }}\right)$. All the curves are for the same system parameters as in Fig. 2 (i.e., kinesin in vitro from Table I). (b) Detachment rate $Q(F)$ computed from simulations with the same models considered in panel (a) shown together with the expected detachment rate $P_{d}\left(F(\right.$ ext $)$ ). (c) Velocity as a function of $\tau_{m}$ for fixed $F_{\text {ext }}=4.8 \mathrm{pN}$ for the same calculations as in panel (a), together with the expected result $v_{\text {obs }}\left(F_{\text {ext }}\right)$. (d) Detachment rate as a function of $\tau_{m}$ for fixed $F_{\text {ext }}=4.8 \mathrm{pN}$ together with the expected result $P_{d}\left(F_{\text {ext }}\right)$.

Table I as the expected results to be reproduced with the simulations. However, the same could be done in principle for any force-velocity and force-detachment relations obtained experimentally. More specifically, suppose that an experiment on single-motor cargo transport with imposed external force yields $v_{\text {exp }}\left(F_{\text {ext }}\right)$ and $P_{\text {exp }}\left(F_{\text {ext }}\right)$ as the force-velocity and force-detachment relation, respectively. Then, the appropriate definitions for the stepping and detachment rates of the motors within the TAF model are $P_{s}\left(\tilde{F}_{i}\right)=v_{\exp }\left(\tilde{F}_{i}\right) / \Delta x$ and $P_{d}\left(\tilde{F}_{i}\right)=P_{\exp }\left(\tilde{F}_{i}\right)$.

\section{Influence of the viscous drag}

In order to study the influence of the viscosity on the SLMC, ZT, and TAF models, we performed simulations for different values of $n_{w}$ at fixed $R=500 \mathrm{~nm}$. One of the main points here is to see if the differences between the models remain relevant at intermediate or large viscosities, since in previous sections we have only analyzed the cases $n_{w}=1$ and $n_{w}=10$.

Note that the viscosity affects the viscous force in the three models and also the noise terms in the SLMC and TAF models. Recall that a change in the medium viscosity is equivalent to a change in the particle size, since both parameters enter only through the combination $\gamma=6 \pi R n_{w} \eta_{w}$.

In Fig. 6(a) we show the force-velocity relations for varying $n_{w}$ for the three models, and the approximate analytic solution explained in Appendix C. As expected, in all the cases the growth of $n_{w}$ leads to a decreasing of the velocity below stall. However, the SLMC model is more sensitive to the growth of $n_{w}$ than the ZT and TAF models. These two latter models are essentially coincident below stall and agree also with the analytical approximation. Concerning the behavior at stall, we find that for the SLMC and ZT models, the stall force does not change with the viscosity. In contrast, for the TAF model it increases from $5 \mathrm{pN}$ at $n_{w}=1$ to $\sim 5.7 \mathrm{pN}$ at $n_{w}=100$ and $\sim 5.9 \mathrm{pN}$ at $n_{w}=300$. Interestingly, in [42] the authors have recently predicted a growth of the stall force with the effective viscosity using a more detailed model for kinesin. The relation between stall forces and viscosity deserves a deep analysis since there is a great dispersion of stall values found in experiments performed in different conditions both for kinesin and dynein (see [6] and the discussion and references in [25]).

Figure 6(b) analyzes the dependence of the calculated detachment rate on the external force for a system with $n_{w}=100$. For the three models, the detachment rate at zero force shifts from the value $P_{d}(0)$ obtained at $n_{w}=1$ to a value close to $P_{d}\left(\gamma v_{0}\right)$ (see indication in the figure). This could be expected since the viscosity increases the effective load acting on the motor. Regarding the behavior at forces close to stall, we see that for the SLMC and ZT models the detachment curves at $F_{\text {ext }} \gtrsim 3$ found for $n_{w}=100$ coincide with those for $n_{w}=1$ [compare with Fig. 2(b) for the SLMC]. In contrast, for the TAF model the viscosity affects the detachment rate around stall, since the abrupt change found for $n_{w}=1$ becomes smoother with increasing viscosity. Finally, for the sake of completeness 6(c) show the dependence of the velocity on $n_{w}$ for the three models at a fixed value of the force. 

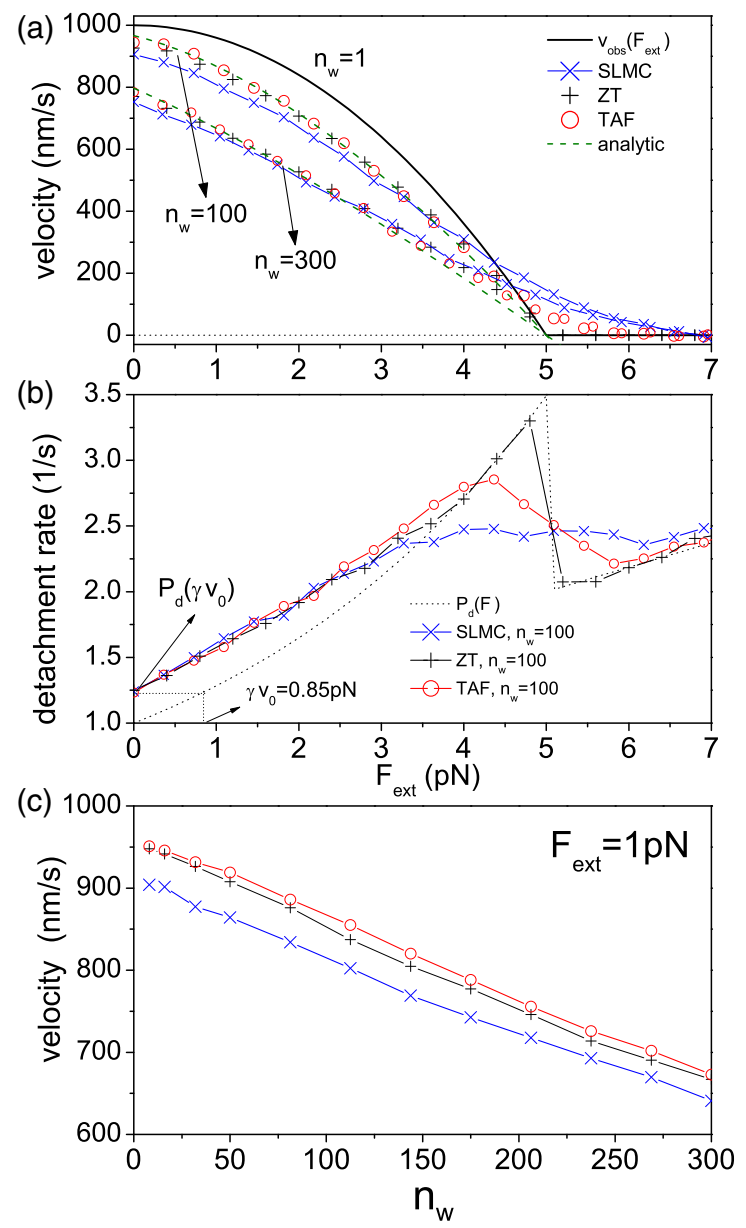

FIG. 6. Influence of the viscosity. Panel (a) shows the forcevelocity curves calculated with the three models considering $n_{w}=$ 100 and $n_{w}=300$ with all the rest of the parameters and probabilities defined as for the in vitro kinesin system studied in Fig. 2. For the sake of comparison, we also show the function $v_{\mathrm{obs}}\left(F_{\mathrm{ext}}\right)$ which coincides with the results for $n_{w}=1$ for the TZ and TAF models [see Fig. 2(a)]. The analytical prediction obtained in Appendix $\mathrm{C}$ is also shown. Panel (b) shows $Q\left(F_{\text {ext }}\right)$ computed for the case $n_{w}=100$ with the three models, together with the assumed $P_{d}(F)$ which coincides with the expected $Q\left(F_{\text {ext }}\right)$ for $n_{w}=1$ [see Fig. 2(b)]. Panel (c) shows the velocity as a function of $n_{w}$ for the SLMC, ZT, and TAF models at fixed $F_{\text {ext }}=1 \mathrm{pN}$. The same line styles as in panels (a) and (b) are used.

As an answer to the question posted at the beginning of the section, our results indicate that the the differences between the models remain relevant at large viscosities, specially for forces close to stall. For small forces, the differences between the models decrease with the viscosity, but they are still relevant at $n_{w}=300$ [see Fig. 6(c)].

\section{Trajectories and mean square displacements}

As mentioned in the Introduction, the analysis of fluctuations of the organelle position in different contexts can be of relevance for the characterization of the properties of the cellular medium and of the motor linkers as well. Thus it is interesting to compare the fluctuation's dynamics in the different models. Here we analyze the fluctuations of the cargo position during single-motor transport for the SLMC, TAF, and ZT models, and also for the two-dimensional (2D) model introduced in Appendix B. As we will show, the latter model provides more realistic results at vanishing external forces.

In order to characterize the fluctuations we analyze the mean square displacement (MSD) of the cargo as a function of the lag time $\Delta t[26,32,33]$ in the range $10^{-5}-5 \mathrm{~s}$. To calculate the MSD, we perform simulations with a fixed run time equal to ten seconds. Thus these simulations are not stopped if the motor detaches before such a run time. Instead, the motor is reattached immediately at the microtubule site closest to $x+x_{r}$. Another possible way to obtain long trajectories for single motor transport could be to forbid detachment. However this could lead to artificial situations with high tension in the motor-spring that may change the statistics of the fluctuations, at least for large external force or large viscosity.

For a trajectory $x(t)$ sampled at times $t_{i}=i \delta t$ with $i=$ $0,1,2, \ldots, N$, the MSD is computed as [32]

$$
[\Delta x(\Delta t)]^{2}=\frac{1}{N-\Delta t / \delta t} \sum_{i=1}^{N-\Delta t / \delta t}\left(x\left(t_{i}+\Delta t\right)-x\left(t_{i}\right)\right)^{2} .
$$

Then, we average over trajectories to get the ensemble averaged mean square displacement $\rho(\Delta t)=\left\langle[\Delta x(\Delta t)]^{2}\right\rangle$. For the sake of simplicity we refer to $\rho(\Delta t)$ as the MSD. We always consider $\delta t=10^{-5} \mathrm{~s}$.

For the 2D model, the MSD is computed considering only the displacements along the $x$ direction. This means using the same formula given in Eq. (9). As shown in [26], during motor driven transport, the displacements on the direction perpendicular to the microtubule are negligible when compared with those along the microtubule for lag times larger than the time between motor steps (typically $\sim 10^{-2} \mathrm{~s}$ ). Thus the MSD in two dimensions coincide with that of the $x$ coordinate at intermediate and large lag times. Moreover, at small lag times, the mean displacements along the $x$ and $y$ directions are essentially the same so that the MSD in two dimensions is approximately twice that of the $x$ coordinate [26].

As before mentioned, here we focus on the analysis of the MSD for single-motor transport to point out some relevant features. It is worth noting that the MSD for multiple motor transport has been systematically studied in [26]. Figure 7 shows typical cargo trajectories and MSD results for various force and viscosity conditions considering the parameters for kinesin in vitro. Given that the trajectories from the SLMC and TAF models are indistinguishable at a first glance, TAF trajectories are not shown. The similarity between the fluctuations in the SLMC and TAF models is reflected by the fact that the MSD curves for these two models coincide.

Figure 7(a) shows trajectories for $n_{w}=1$ and $F_{\text {ext }}=3 \mathrm{pN}$ (i.e., small viscosity and an intermediate load force below stall). For the SLMC (and TAF) the fluctuations are small enough to appreciate clearly the $8 \mathrm{~nm}$ motor steps. This is compatible with what is usually found in in vitro experiments [40]. Meanwhile, the trajectory for the ZT model shows no fast fluctuations and the cargo advance in discrete steps. This expected unrealistic output of the ZT model is clearly reflected by the MSD results shown in Fig. 7(b). We see that for $\Delta t<$ $10^{-2} \mathrm{~s}$ the SLMC and TAF models indicate a very slowly varying MSD in the range $10 \mathrm{~nm}^{2} \lesssim \rho \lesssim 50 \mathrm{~nm}^{2}$, while the 

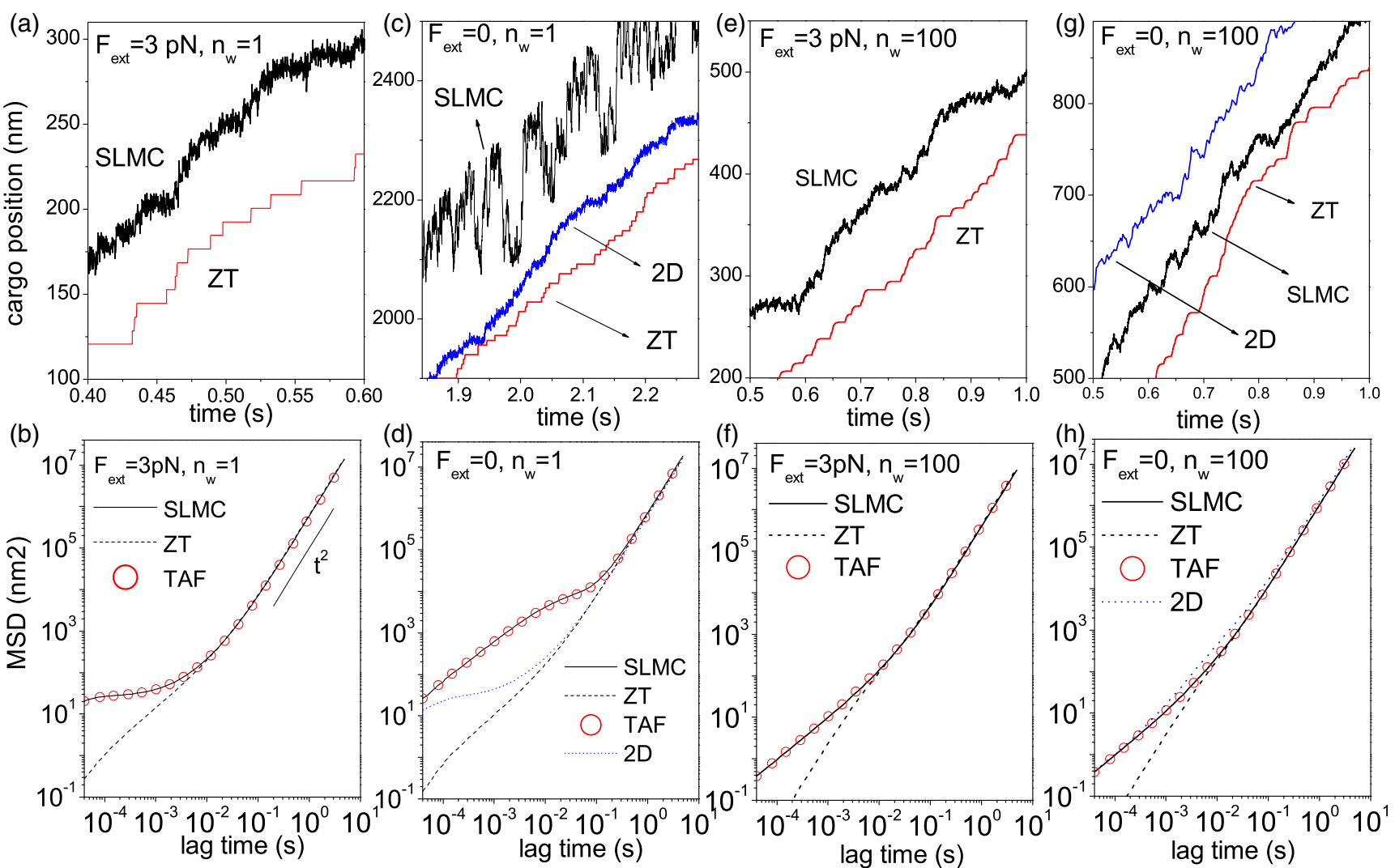

FIG. 7. Trajectories and MSD. Cargo trajectories (top panels) and MSD as function of the lag time (bottom panels) for various force and viscosity conditions and models. See indications on each panel. Except for the values of $n_{w}$, in all the cases the parameters are those for kinesin in vitro indicated in Table I. The curves named as $2 \mathrm{D}$ correspond to the bidimensional model introduced in Appendix B.

ZT model exhibit much smaller fluctuations. On the other hand, at lag times larger than $\Delta t>10^{-2} \mathrm{~s}$, all the three models agree in the prediction of a ballistic behavior.

Figure 7(c) and Fig. 7(d) analyze the same system as Figs. 7(a) and 7(b) but considering $F_{\text {ext }}=0$. A first remarkable observation is that the trajectory for the SLMC model has huge fluctuations with amplitude of order $200 \mathrm{~nm}$. This contrasts with experiments and with the SLMC simulations for $F_{\text {ext }}>0$ [see Fig. 7(a)]. The result can be attributed to the effect of the particular form assumed for the motor-cargo interaction combined with the one-dimensional character of the model. Note that the motor-cargo interaction is actually associated to a symmetric potential well which is constant (and minimum) in a region of width $2 x_{r}=220 \mathrm{~nm}$ and then it grows quadratically. Thus, in the absence of an external force, the cargo position is expected to fluctuate almost freely within the region where the potential is flat. In contrast, for positive enough $F_{\text {ext }}$, the cargo is pulled away from the motor position $x_{i}$ and is confined close to $x_{i}-x_{r}$. The problem at $F_{\text {ext }}=0$ is associated to the one-dimensional character of the model. Note that, in the experiments, the interaction potential is expected to act along the direction of the motor stalk in three dimensions and not necessarily along the microtubule. A correct description of the fluctuations at small loads require thus a higher dimensional modeling. In fact, the two-dimensional model introduced in Appendix B solves the problem. It produces the trajectory shown in Fig. 7(c) which has an appropriate level of fluctuations enabling for the identification of the $8 \mathrm{~nm}$ steps and MSD values of the order of $10 \mathrm{~nm}^{2}$ at small lag times [Fig. 7(d)]. We believe that three-dimensional models as those considered in [23,24] would also provide correct and even better characterizations of the fast fluctuations of the cargo position.

Figures 7(e)-7(h) repeat the results of panels (a)-(d) but considering $n_{w}=100$ instead of $n_{w}=1$. For $F_{\text {ext }}=3$, the trajectories for the SLMC model for $n_{w}=100$ are more wrinkled in the scale of $\Delta t \sim 0.1 \mathrm{~s}$ than those for $n_{w}=1$, and the $8 \mathrm{~nm}$ steps are hardly observable. Meanwhile, the large fluctuations observed for the SLMC at $F_{\text {ext }}=0$ for $n_{w}=1$ are suppressed by the large viscous force. In fact, the $F_{\text {ext }}=3$ and $F_{\text {ext }}=0$ trajectories have quite similar characteristics. The 2D model does not lead to any significant advantage. Finally, the ZT-model trajectories for $n_{w}=100$ show less steep $8 \mathrm{~nm}$ steps than those for $n_{w}=1$. This obeys to the largest relaxation time $\gamma / k$ associated to the motor-cargo linker. As for the $n_{w}=1$ case, the MSD curves for the SLMC and TAF models coincide, while the ZT model gives smaller values at small lag times.

\section{E. Results for multiple motor transport}

One of the main aims of the development of the LMC models is the analysis and interpretation of multiple-motor transport by coupling individual motors dynamics based on single-motor experiments. Here we compare the results of the SLMC, ZT, and TAF models for several relevant examples of multiple motor transport. 

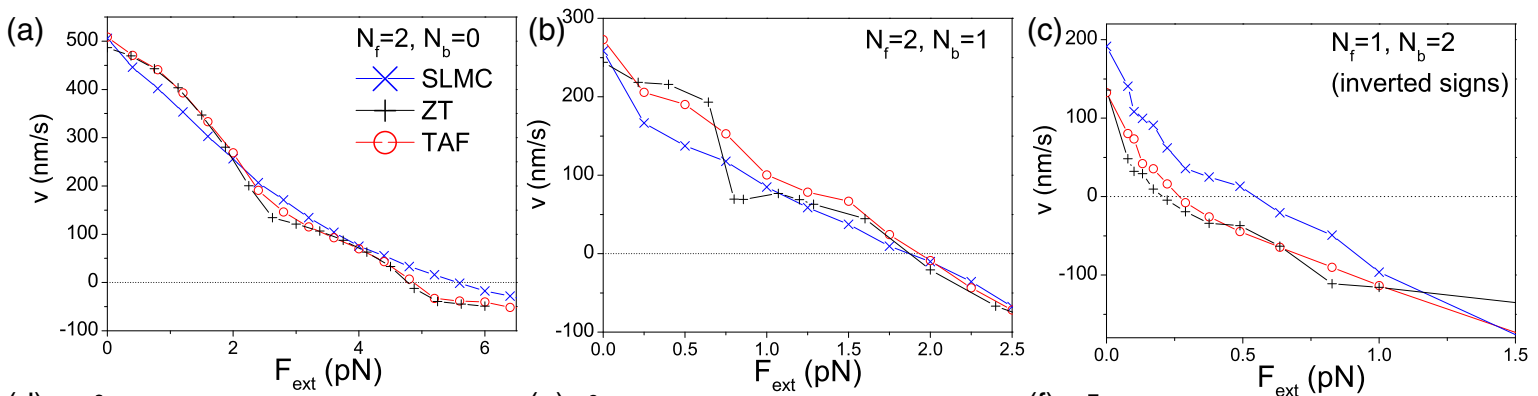

(d)

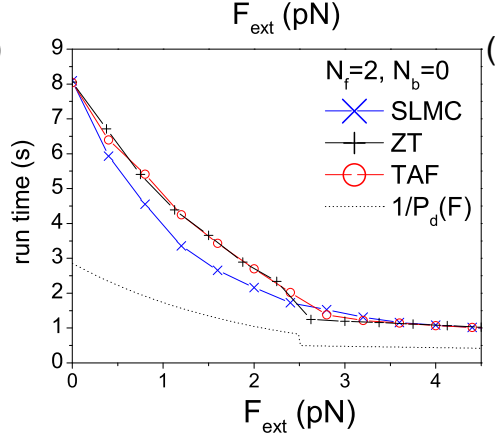

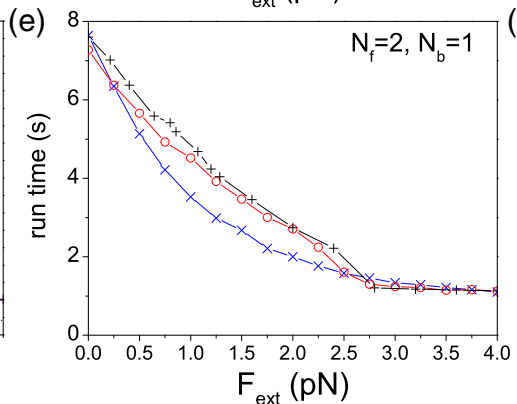

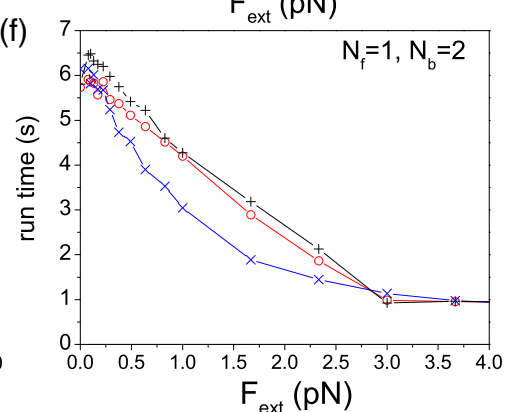

FIG. 8. Multiple motor transport with $N_{f} \neq N_{b}$. Mean velocities (top panels) and mean run times (bottom panels) as functions of the external force calculated from simulations with the different models and motor numbers. For forward and backward motors we consider the parameters and probability functions given in Table I for kinesin in vivo and dynein in vivo, respectively. The velocity results for the case $N_{b}>N_{f}$ shown in panel (c) have the signs inverted so that a positive velocity indicates transport towards the minus end.

The simulations for multiple motors are performed in a completely analogous way to those for single motors. At initial condition, each motor is attached in one of the sites $x_{j}=j \Delta x$ satisfying $\left|x-x_{j}\right|<x_{r}$. Each realization ends when all the motors have been detached.

An important quantity to analyze is the ratio between the stall force for transport by two kinesins to that of a single kinesin. This has been experimentally determined as close to 1.87 (see [4] and references therein). Our calculations with the SLMC model yield the value 1.47 both for the in vitro and in vivo cases, while the TAF model gives 1.83 and 1.93 , respectively, i.e., much closer to the experimental result. These values were obtained from the force-velocity relations found in simulations with the parameters indicated in Table I. The stall force considered for each case is the one leading to zero mean velocity. The results for the ratio do not change significantly if we consider the condition of run-length equal to $8 \mathrm{~nm}$ for defining the stall force [4] instead of the condition of zero mean velocity. With this prescription, the SLMC gives 1.52 for kinesin in vitro and 1.45 for in vivo, while the TAF model yields 1.89 and 1.93, respectively. In Ref. [4], using an LMC model with a Monte Carlo algorithm specialized for kinesin with no time averaging, the authors found the ratio as equal to 1.77 when considering the run length criterion and equal to 2 for the zero mean-velocity criterion.

Now we present more general results for multiple motor transport. In all the cases we consider the parameters and probabilities given for kinesin in vivo and dynein in vivo in Table I. Note that the parameters for kinesin and dynein are similar but not equal. Thus, even for $N_{f}=N_{b}$, the dynamics is not expected to be perfectly symmetric. It is worth remarking that our main purpose here is to show how the differences between the SLMC, TAF, and ZT models affect the multiple motor cases, and not to analyze any particular experiment reported.

First we consider situations with $N_{f} \neq N_{b}$, and we calculate the mean velocity and mean run time as functions of the external force $F_{\text {ext }}$ in a completely analogous way to what is done for single motor transport. In all the cases, $F_{\text {ext }}$ is assumed to point against the motion of the motor species with the larger number of motors. This means to the minus end for $N_{f}>N_{b}$ and to the plus end for $N_{b}>N_{f}$. Figure 8 shows the results corresponding to the systems with $\left(N_{f}, N_{b}\right)=(2,0),(2,1)$, and $(1,2)$. We see that, in most cases, the TAF and ZT models give very similar results, while the SLMC model departs considerably. The exception corresponds to the velocity results for the case $\left(N_{f}, N_{b}\right)=(2,1)$, in which all the three models differ.

Finally, we turn to cases with $N_{f}=N_{b}$. Figure 9(a) shows typical trajectories for $N_{f}=N_{b}=2$ calculated with the SLMC model. Bidirectional motion $[5,10]$ with forward and backward stages and also intermediate pauses associated to the tug-of-war effect $[5,10,14]$ are observed. Trajectories for the TAF model are indistinguishable at first glance from those of the SLMC. In our analysis, we focus on the calculation of the mean maximal excursion to each polarity and the mean final position as functions of $n_{w}$. These quantities are defined as follows. First, the forward (backward) mean maximal excursion is the average over realizations of the largest positive (negative) position attained by the cargo during a run. Meanwhile, the mean final position is the average on realization of the position at which complete detachment occurs. In Figs. 9(b) to 9(e) we show the results for $N_{b}=N_{f}=2$ and $N_{b}=N_{f}=3$. We see that, although kinesin results always the stronger species, the three models differ in their quantitative predictions. Moreover, it can be seen 

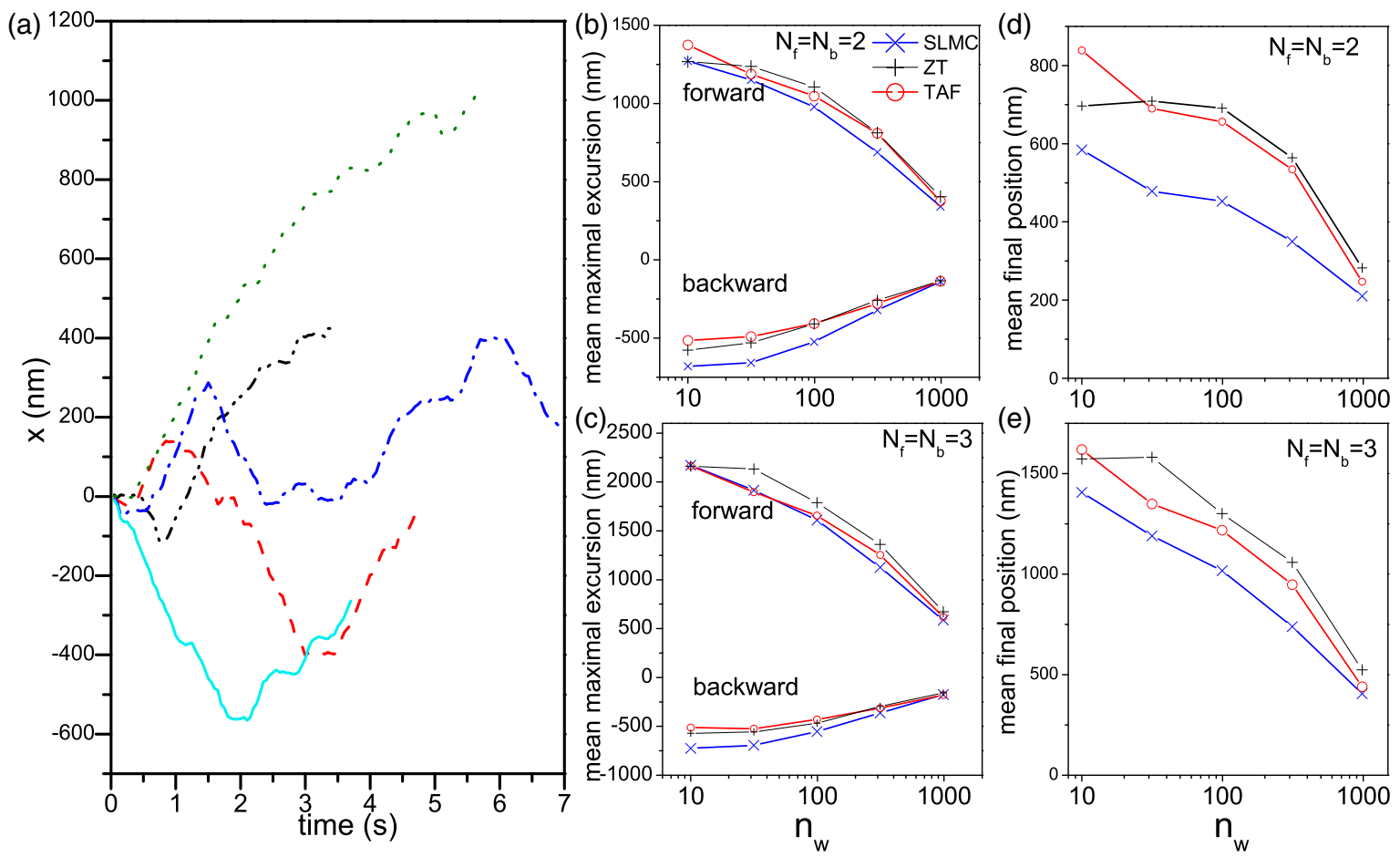

FIG. 9. Multiple motor transport with $N_{f}=N_{b}$. (a) Typical trajectories of bidirectional motion obtained with the SLMC model considering $N_{f}=N_{b}=2$. The other panels show results for the mean maximal excursions and mean final position as functions of $n_{w}$ computed for the cases $N_{b}=N_{f}=2$ [panels (b) and (d)] and $N_{b}=N_{f}=3$ [panels (c) and (e)] considering the different motor models. In all the cases, the parameters used for forward and backward motors are those given in Table I for kinesin in vivo and dynein in vivo, respectively, with the exception of the value of $n_{w}$ indicated in panels (b) to (e).

that, excepting for some of the quantities computed at small viscosity, the results from the ZT and TAF models are closer to each other than those from the SLMC.

Altogether, our results for multiple motors show that the differences between the single-motor models propagate to the case of multiple motor transport. The fact that the TAF and ZT models predict the correct force-velocity and force-detachment relations for single motors, and also the correct ratio between the two-motor and single motor stall forces, suggests that these models are more reliable than the SLMC model for general multiple motor situations.

\section{F. Other LMC models: Parameter fitting and models with back steps}

Here we go back to single motor transport and analyze the relation of our results with previous studies on LMC models different from the SLMC.

Our analysis of the SLMC showed that the calculated stall force for single motors is larger than the parameter $F_{0}$ defined in Table I as the local force leading to zero stepping probability. This also occurs in other LMC models which consider the motor's stepping and detachment rates as dependent on the instantaneous forces (i.e., with no time averaging). For instance, in [4] the authors consider an LMC model for kinesin with a stepping algorithm which includes two instances of the chemical cycle of kinesin and two mechanisms for detachment. In that model, the stepping probability includes a global factor $\left(1-\left(F / F_{0}\right)^{w}\right)$ just as in the SLMC, and the parameter $F_{0}$ is actually tuned to values smaller than stall to observe the correct stall force. For example, the authors use $F_{0}=5.1 \mathrm{pN}$ to get a stall value of $5.7 \mathrm{pN}$. The factor $\left(1-\left(F / F_{0}\right)^{w}\right)$ is interpreted as a coupling efficiency between ATP hydrolysis and mechanical stepping.

Within the SLMC model, one could also think on the possibility of fitting the parameter $F_{0}$ to a value smaller than stall in order to get the correct stall force in simulations. However, a problem would arise with the detachment probability, at least for the case of nonmonotonous or discontinuous detachment rates. In fact, there is not an obvious way for defining $P_{d}(F)$ in order to get the expected $Q\left(F_{\text {ext }}\right)$ for the cases analyzed in Figs. 2(b), 3(b), and 3(d) without time averaging. The fluctuations of the force always seem to flatten the nonmonotonic behaviors. We have in fact attempted several definitions of $P_{d}(F)$ to reproduce the results in Fig. 2(b) within the SLMC with no success. Finally, this failure led us to propose the TAF model to solve the problem.

A relevant question that arises is whether the consideration of time-averaged forces in the motor dynamics may be useful to improve the performance of other LMC models different from the SLMC. In particular, the case of models with motor back-stepping [14,22] is of interest, since in such models the behavior at stall is qualitatively different from that in the SLMC. While in the SLMC the stall condition occurs when the motor-stepping probability tends to zero, in the model with back steps it occurs when forward and backward steps becomes equally probable. Moreover, the models with back steps reproduces the processive backward motion observed at superstall conditions [22]. To answer the question posed, we analyze the model with back steps introduced in Appendix B 
which was developed originally in [14,22], and we also implement the corresponding version with time averaged forces (see in Appendix B). The following definition for $v_{\text {obs }}\left(F_{\text {ext }}\right)$ is considered:

$$
v_{\text {obs }}\left(F_{\text {ext }}\right)= \begin{cases}v_{0}, & F_{\text {ext }}<0, \\ v_{0}\left[1-\left(F / F_{0}\right)^{2}\right], & 0 \leqslant F_{\text {ext }} \leqslant F_{0}, \\ -50 \mathrm{~nm} / \mathrm{s} \tanh \left[1.5(\mathrm{pN})^{-1}\left(F-F_{0}\right)\right], & F_{\text {ext }}>F_{0},\end{cases}
$$

which includes slow backward motion at superstall $\left(F_{\text {ext }}>\right.$ $\left.F_{0}\right)$. We consider $v_{0}=1000 \mathrm{~nm} / \mathrm{s}$ and $F_{0}=5 \mathrm{pN}$. As in our previous analysis for the SLMC and TAF models using Eq. (8), the formula in Eq. (10) is the force-velocity relation that we want to reproduce with the simulations at small viscosity. In Fig. 10(a) we show $v_{\text {obs }}\left(F_{\text {ext }}\right)$ as defined in Eq. (10) together with the velocity results from simulations performed with the backsteps model with and without time averaged forces. We see that the model with time averaged forces leads to a much better agreement with $v_{\text {obs }}\left(F_{\text {ext }}\right)$. In particular, note that the model with no time averaging overestimates the stall force as happened with the SLMC. The results are thus completely analogous to those found in Fig. 4 when comparing the SLMC and TAF models. Finally, Fig. 10(b) shows the velocity
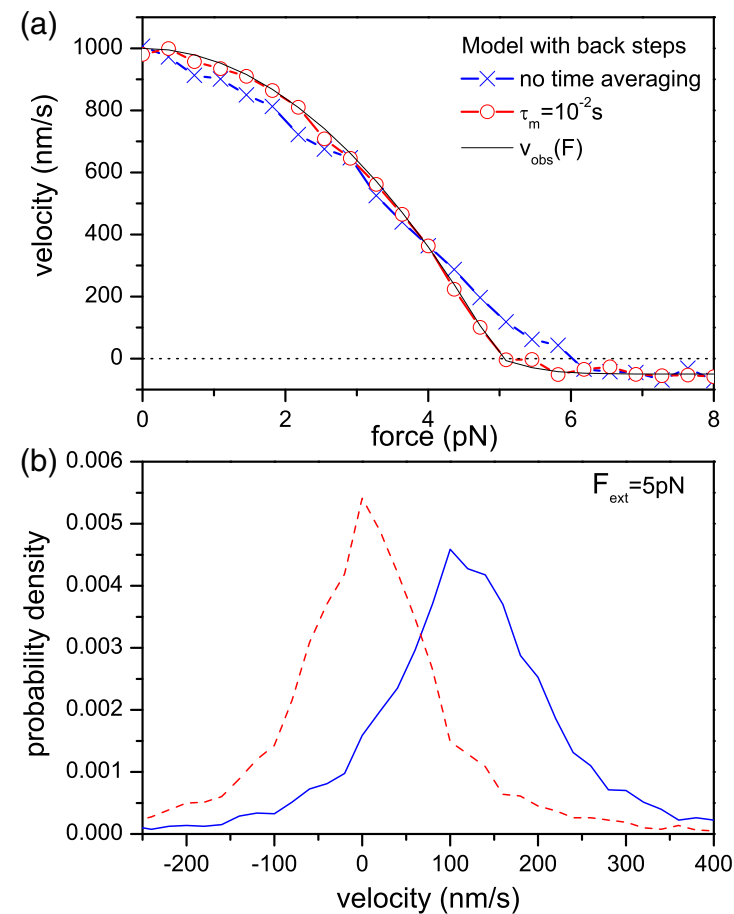

FIG. 10. Results for the model with back steps with and without the time averaging procedure. (a) Mean velocity as a function of the external force computed from simulations with the model with back steps introduced in Appendix B. Results for motor models with and without time averaging of the forces. For the sake of comparison, the expected velocity $v_{\text {obs }}$ given in Eq. (10) is also shown. Except for the definition of the stepping probability, the parameters are those given in Table I for kinesin in vitro. (b) Velocity distributions for the same systems analyzed in panel (a) for fixed $F_{\text {ext }}=5 \mathrm{pN}$. The solid line corresponds to the back-steps model with no time averaging, while the dashed line is for the case $\tau_{m}=0.01 \mathrm{~s}$. distributions calculated for the backsteps model with and without time averaging for $F_{\text {ext }}=5 \mathrm{pN}$ (i.e., stall condition). Again, the conclusions are analogous to those found when comparing the SLMC and TAF models in the analysis done in Figs. 4(b) and 4(e). These results indicate that the consideration of time averaged forces for ruling the motor dynamics is useful not only for the SLMC scheme but also for more general LMC frameworks.

\section{DISCUSSION AND CONCLUSIONS}

We have analyzed the performance of one-dimensional models for microtubule transport which combine a continuous dynamics for the cargo with a stochastic stepping dynamics for the motors. In particular, we have introduced a model for the motor dynamics which was referred to as the time-averaged forces model (TAF) and compared it to a standard LangevinMonte Carlo model (SLMC) previously considered in various publications, and to a zero temperature (ZT) version of the SLMC.

The consideration of time-averaged forces for ruling the motor dynamics in the TAF model implies the introduction of memory on the history of the force felt by the motors. Due to the fact that the various stages of the chemical cycles of the motors depend on the force in different ways which are not described in detail in our model, we have proposed the time between steps as the appropriate time scale for averaging. Our results and further mathematical analysis in the section "Robustness of the TAF model against varying $\tau_{m}$ " support this consideration.

Our main results show that the TAF and ZT models perform significantly better than the SLMC in the reproduction of the force-velocity and force-detachment relations for single motors. Moreover, they give a much more exact prediction for the stall force of two motors than the SLMC. On the other hand, given that the ZT model does not consider thermal noise, only the TAF and SLMC models can produce reasonable descriptions of the rapid fluctuations of the particle position. Thus, among the studied models, the TAF arise as the best option for interpreting experiments in which not only the global properties of the motion but also the fluctuations are of interest. Regarding this, we have also shown that, depending on the external forces and viscosities, the one-dimensional modeling can have some limitations and twoor three-dimensional formulations may be needed.

The results presented for the model with motor backstepping indicate that the consideration of time averaged forces for the motor dynamics is useful for improving the performance of more general Langevin-Monte Carlo schemes. 
The one-dimensional models analyzed in this paper consider the nonlinear function given in Eq. (3) for modeling the elastic properties of the motors. This assumption was also introduced in $[4,6,10,14,23,26,43]$ and other works. As we have shown, the approximation allows for instance for the prediction of the correct value of the stall force for two motors within the TAF model, but it does not produce a good description of the fluctuations of the cargo position at small loads. Concerning this, we have also shown that the inclusion of an additional repulsive interaction at small distances in a two dimensional framework improves the results. Moreover, the studies in $[4,14]$, among others, indicate that the results for multiple-motor transport depend on the values of the stiffness constants. This all strongly suggests that the validity of the approximation is limited and that further studies on the influence of the elastic properties of the motors are needed.

Concerning multiple-motor transport, we have seen that the simulation's results are rather sensible to the differences on the single motor modeling. Note that for some of the quantities analyzed in Figs. 8 and 9 the predictions of the ZT and TAF do not coincide, even though these two models agree in reproducing the correct force-velocities relations, and also the correct two motor's stall force. This indicates that special care should be taken for interpreting multiplemotor experiments. Verifications using several models could be necessary sometimes. Although our analysis has focused on the mean velocities, the run lengths and the maximal excursions, other quantities relevant for the characterization of multiple-motor transport such as pause times [6] and distributions of segmental velocities $[6,25]$ are also expected to be affected by the details of the modeling.

As pointed out in the Introduction, the analysis of the fluctuations of the motion of organelles in vivo is of relevance for the characterization of the viscoelastic properties of the cytoplasm and the elastic properties of the motor linkers as well. The study of the interplay between thermal and active transport taking into account the rheological properties of the cellular medium is on its beginnings [26,27,43], and needs for motor models which can correctly reproduce the results for the mean velocities and run-times in a noisy environment. We believe that the TAF model here developed is a step forward in this direction.

\section{ACKNOWLEDGMENTS}

The author thanks A. B. Amaya and G. Mato for valuable comments, and acknowledges support from CONICET (under Grant No. PIP 11220110100310) and from CNEA, both Argentinian agencies.

\section{APPENDIX A: ALGORITHMS FOR THE TIME EVOLUTION OF THE SLMC, ZT, AND TAF MODELS}

In the SLMC model, the evolution of the cargo-motor system is computed as follows. The time is discretized in steps of duration $d t$ which must be considered much smaller than the relaxation time of the motor springs $\gamma / k$. Typically we consider $d t \sim 10^{-6} \mathrm{~s}$ or lower, depending on the viscosity. At each time step, given the cargo position $x(t)$ and the motor positions $x_{i}(t)\left(i=1, \ldots, N_{b}+N_{f}\right)$, the forces $F_{i}(t)$ are computed with the formulas given in the main text. Then, the cargo position at $t+d t$ is calculated using the standard discretization of the Langevin equation

$$
x(t+d t)=x(t)+\left[-F_{\mathrm{ext}}+\sum_{i=1}^{N_{f}+N_{b}} F_{i}\right] \frac{d t}{\gamma}+\sqrt{\frac{2 k_{B} T d t}{\gamma} g .}
$$

Here, $g$ is a random number (different for each time step) taken from a Gaussian distribution with zero mean and unitary variance. After computing $x(t+d t)$, the following algorithm steps are implemented for each attached motor. First, the detachment probability $p_{d}=P_{d}\left(F_{i}(t)\right) d t$ for the present time step is computed. Then, a random number $r_{1}$ uniformly distributed between 0 and 1 is computed. If $r_{1}<p_{d}$, the motor is marked as detached at time $t+d t$. If the motor does not result detached, the probability $p_{s}=P_{s}\left(F_{i}(t)\right) d t$ is computed and an analogous test with a new random number $r_{2}$ is performed to evaluate the possibility of stepping. If the step is approved, we set $x_{i}(t+d t)=x_{i}(t)+\Delta x$ or $x_{i}(t+d t)=$ $x_{i}(t)-\Delta x$ depending on whether the $i$ th motor is a forward or a backward motor. If not, we set $x_{i}(t+d t)=x_{i}(t)$. Finally, each detached motor is allowed to attach to the microtubule with a probability $\Pi d t$ (not the motors marked in the present time step but only those that were already detached at time $t$ ). The attachment occurs with equal probability in any of the sites $x_{j}=j \Delta x$ with integer $j$ satisfying $\left|x(t)-x_{j}\right|<x_{r}$ (i.e., with the motor-linker relaxed).

The algorithm for the ZT model is the same as that for the SLMC but we take $k_{B} T=0$ (or equivalently $g=0$ ) in Eq. (A1) for all the time steps.

Finally, the algorithm for computing the time evolution of the TAF model is the following. At each time step, given the values of $x(t), x_{i}(t)$, and $\tilde{F}_{i}(t)$, we first compute the forces $F_{i}(t)$ as in the SLMC model. Then, we compute $x(t+d t)$ using Eq. (A1). After that, using the values of $\tilde{F}_{i}(t)$ and the calculated $F_{i}(t)$, we compute $\tilde{F}_{i}(t+d t)$ as

$$
\tilde{F}_{i}(t+d t)=\tilde{F}_{i}(t)-\frac{d t}{\tau_{m}}\left[\tilde{F}_{i}(t)-F_{i}(t)\right] .
$$

This is just the Euler approximation for Eq. (5). Finally, the motor positions are evolved with the same Monte Carlo algorithm explained for the SLMC, but using the timeaveraged forces $\tilde{F}_{i}(t)$ instead of $F_{i}(t)$ for defining the stepping and detachment probability.

\section{APPENDIX B: OTHER LMC MODELS}

\section{Model with back steps}

We consider an LMC model which includes motor backstepping. The dynamics of the cargo is given by Eq. (1). The algorithm for motor stepping is defined as in [14,22] through two quantities that can be measured in experiments [40]. Namely, the dwell time $\tau_{d}\left(F_{i}\right)$ (i.e., the mean time between steps) and the ratio between forward and backward steps $R_{f b}\left(F_{i}\right)$.

At each time step, after evaluating the possibility of detachment as in the SLMC model, the motor performs an $8 \mathrm{~nm}$ step with probability $d t / \tau_{d}\left(F_{i}\right)$. The step is defined to be a forward step with probability $R_{f b}(F v) /\left(R_{f b}\left(F_{i}\right)+1\right)$, while it 
is defined as a backward step with probability $1 /\left(R_{f b}\left(F_{i}\right)+1\right)$. For such a motor model, the expected velocity at constant load force $F_{i}$ (i.e., without cargo and thermal fluctuations) is

$$
v_{E}\left(F_{i}\right)=\left(\Delta x / \tau_{d}\right)\left(R_{f b}\left(F_{i}\right)-1\right) /\left(R_{f b}\left(F_{i}\right)+1\right) .
$$

We consider $R_{f b}\left(F_{i}\right)$ defined as in [22]

$$
R_{f b}\left(F_{i}\right)=1000 \exp \left[-\log (1000) F_{i} / F_{0}\right] .
$$

Note that for $F_{i}=F_{0}$ we get $R_{f b}=1$.

Now, assuming for $v_{\text {obs }}\left(F_{\text {ext }}\right)$ the formula given in Eq. (10) of the main text (which is negative at superstall conditions) and equating $v_{E}\left(F_{i}\right)$ to $v_{\text {obs }}\left(F_{i}\right)[14,22]$ we get

$$
\tau_{d}\left(F_{i}\right)=\frac{\left(R_{f b}\left(F_{i}\right)-1\right) \Delta x}{\left(1+R_{f b}\left(F_{i}\right)\right) v_{\mathrm{obs}}\left(F_{i}\right)} .
$$

Recall that, as indicated in the main text, $v_{\text {obs }}\left(F_{\text {ext }}\right)$ represents the results that we want to reproduce with our simulations.

Finally, by considering the time averaged forces $\tilde{F}_{i}(t)$ instead of $F_{i}$ for defining the motor dynamics [see Eqs. (4) and (5)], we arrive at a model with back steps and time averaged forces which depends on the parameter $\tau_{m}$.

\section{Two-dimensional model for cargo transport along a microtubule}

In order to solve the problem of large fluctuations observed in one-dimensional schemes for vanishing external force (see Fig. 7), we introduce a two-dimensional model (2D) for the cargo-motor system. This 2D scheme was previously considered in [26] although for a viscoelastic medium.

We allow the organelle to move in two dimensions by considering the 2D Langevin equation given by

$$
\gamma \dot{\vec{r}}=-\vec{F}_{\text {ext }}+\vec{F}+\vec{\xi},
$$

where now the position of the cargo $\vec{r}(t)=(x(t), y(t))$, the external force $\vec{F}_{\text {ext }}=\left(F_{\text {ext }}, 0\right)$, the motor force $\vec{F}=\left(F_{x}, F_{y}\right)$, and the thermal noise $\vec{\xi}$ are vectorial quantities. Concerning the model for the motors motion, we still consider a one dimensional filament along the $x$ axis so that the allowed positions for the motor heads on the filaments are of the form $\vec{r}=(j \Delta x, 0)$ with integer $j$ and $\Delta x=8 \mathrm{~nm}$ as before. The force $\vec{F}$ exerted on the cargo (at position $\vec{r}$ ) by the motor at position $\vec{r}_{i}$ is calculated as $\vec{F}=-k_{\text {rep }}\left(\left|\vec{r}-\vec{r}_{i}\right|-r_{0}\right) \hat{n}$ for $\left|\vec{r}-\vec{r}_{i}\right| \leqslant r_{0}$ and $\vec{F}=-k\left(\left|\vec{r}-\vec{r}_{i}\right|-r_{0}\right) \hat{n}$ for $\left|\vec{r}-\vec{r}_{i}\right|>r_{0}$. Here $r_{0}=110 \mathrm{~nm}, \hat{n}=\left(\vec{r}-\vec{r}_{i}\right) /\left|\vec{r}-\vec{r}_{i}\right|, k=0.3 \mathrm{pN} / \mathrm{nm}$ is the motor stiffness considered in the one-dimensional model and $k_{\text {rep }}=0.05 \mathrm{pN} / \mathrm{nm}$ the motor repulsion constant at small length. Finally, the motor dynamics along the $x$ axis remains as that for the SLMC but determined only by the $x$ component of $\vec{F}$. This means we consider $P_{s}\left(F_{x}\right)$ and $P_{d}\left(F_{x}\right)$ as the stepping and detachment rates.

Note that the inclusion of a repulsive interaction in a one dimensional model may be meaningless since it would confine the cargo to regions close to either $x=x_{i}+x_{r}$ or to $x=x_{i}-$ $x_{r}$. In contrast, in two dimensions, the repulsive interaction favors the relaxation of the motor spring to the natural length $r_{0}$ while the relative position of the cargo with respect to the motor along the microtubule can change through a trajectory in two dimensions (approximately circular).

\section{APPENDIX C: ANALYTICAL APPROXIMATION FOR THE VELOCITY OF SINGLE-MOTOR TRANSPORT IN VISCOUS MEDIA}

We consider the viscous force as an additional load force acting on the motor and solve the equation

$$
v=v_{0}\left(1-\left(\frac{F+\gamma v}{F_{0}}\right)^{2}\right)
$$

for the variable $v$. Note that this equation is just the definition of $v_{\text {obs }}(F)$ but with the change $F \rightarrow F+\gamma v$. We get two solutions. The relevant (positive) one is

$$
v=\frac{-F_{0}^{2}-2 F \gamma v_{0}+F_{0} \sqrt{F_{0}^{2}+4 F \gamma v_{0}+4 \gamma^{2} v_{0}^{2}}}{2 \gamma^{2} v_{0}} .
$$

[1] J. Howard, Mechanics of Motor Proteins and the Cytoskeleton (Sinauer Associates, Sunderland, MA, 2001); R. Vale, Cell 112, 467 (2003).

[2] M. A. Welte, Curr. Biol. 14, R525 (2004); S. P. Gross, Phys. Biol. 1, R1 (2004).

[3] R. Mallik, A. K. Rai, P. Barak, A. Rai, and A. Kunwar, Trends Cell Biol. 23, 575 (2013).

[4] A. Kunwar, M. Vershinin, J. Xu, and A. P. Gross, Curr. Biol. 18, 1173 (2008).

[5] M. J. I. Müller, S. Klumpp, and R. Lipowsky, Proc. Natl. Acad. Sci. USA 105, 4609 (2008).

[6] A. Kunwar, Proc. Natl. Acad. Sci. USA 108, 18960 (2011).

[7] M. Fu and E. Holzbaur, Trends Cell Biol. 24, 564 (2014).

[8] S. Klumpp and R. Lipowsky, Proc. Natl. Acad. Sci. USA 102, 17284 (2005).

[9] R. Lipowsky et al., Physica E 42, 649 (2010).

[10] A. Kunwar and A. Mogilner, Phys. Biol. 7, 016012 (2010).
[11] F. Berger, C. Keller, S. Klumpp, and R. Lipowsky, Phys. Rev. Lett. 108, 208101 (2012).

[12] F. Berger, C. Keller, S. Klumpp, and R. Lipowsky, Phys. Rev. E 91, 022701 (2015).

[13] W. Nam and B. I. Epureanu, Phys. Rev. E 86, 051916 (2012).

[14] S. Bouzat and S. F. Falo, Phys. Biol. 8, 066010 (2011).

[15] V. Soppina, A. K. Rai, A. J. Ramaiya, P. Barak, and R. Mallik, Proc. Natl. Acad. Sci. USA 106, 19381 (2009).

[16] M. Gazzola, C. J. Burckhardt, B. Bayati, M. Engelke, U. F. Greber, and P. Koumoutsakos, PLoS Comput. Biol. 5, e1000623 (2009).

[17] O. Campas, C. Leduc, P. Bassereau, J. Casademunt, J. F. Joanny, and J. Prost, Biophys. J. 94, 5009 (2008).

[18] D. Chowdhury, A. Schadschneider, and K. Nishinari, Phys. Life Rev. 2, 318 (2005).

[19] D. Gillo, B. Gur, A. Bernheim-Groswasser, and O. Farago, Phys. Rev. E 80, 021929 (2009). 
[20] Y. Zhang, Phys. Rev. E 79, 061918 (2009).

[21] Y. Zhang, Phys. Rev. E 87, 052705 (2013).

[22] S. Bouzat and S. F. Falo, Phys. Biol. 7, 046009 (2010).

[23] C. B. Korn, S. Klumpp, R. Lipowsky, and U. S. Schwarz, J. Chem. Phys. 131, 245107 (2009).

[24] R. P. Erickson, Z. Jia, S. P. Gross, and C. C. Yu, PLoS Comput. Biol. 7, e1002032 (2011).

[25] S. Bouzat, V. Levi, and L. Bruno, Plos One 7, e43599 (2012).

[26] S. Bouzat, Phys. Rev. E 89, 062707 (2014).

[27] I. Goychuk, V. O. Kharchenko, and R. Metzler, PLoS One 9, e91700 (2014).

[28] I. Goychuk, Phys. Rev. E 80, 046125 (2009).

[29] I. Goychuk, Adv. Chem. Phys. 150, 187 (2012).

[30] T. Franosch et al., Nature (London) 478, 85 (2011).

[31] D. Wirtz, Annu. Rev. Biophys. 38, 301 (2009).

[32] E. Bertseva et al., Eur. Phys. J. E 35, 63 (2012).

[33] D. S. Grebenkov, M. Vahabi, E. Bertseva, L. Forró, and S. Jeney, Phys. Rev. E 88, 040701(R) (2013).

[34] L. Bruno, M. Salierno, D. Wetzler, M. Desposito, and V. Levi, PLoS One 6, e18332 (2011).
[35] N. G. Van Kampen, Stochastic Processes in Physics and Chemistry (North-Holland, Amsterdam, 1992).

[36] M. O. Magnasco, Phys. Rev. Lett. 71, 1477 (1993).

[37] F. Jülicher, A. Ajdari, and J. Prost, Rev. Mod. Phys. 69, 1269 (1997).

[38] H. S. Wio, Path Integrals for Stochastic Processes, An Introduction (World Scientific, Singapore, 2013).

[39] B. Shizgal, Spectra Methods in Chemistry and Physics (Springer, New York, 2015).

[40] M. J. Schnitzer, K. Visscher, and S. M. Block, Nat. Cell Biol. 2, 718 (2000); N. J. Carter and R. A. Cross, Nature (London) 435, 308 (2005).

[41] In [23], as the cargo motion is three dimensional, the authors consider the stepping probability as dependent on the projection $F_{m}$ of the motor force on the direction of the microtubule, i.e., $P_{s}\left(F_{m}\right)=v_{\mathrm{obs}}\left(F_{m}\right) / \Delta x$.

[42] E. Zimmermann and U. Seifert, Phys. Rev. E 91, 022709 (2015).

[43] W. Nam and B. I. Epureanu, J. Phys.: Condens. Matter 24, 375103 (2012). 